\title{
Emergent Dynamics of Fast Ripples in the Epileptic Hippocampus
}

\author{
Jose M. Ibarz, ${ }^{2 \star}$ Guglielmo Foffani, ${ }^{3 *}$ Elena Cid, ${ }^{1}$ Marion Inostroza, ${ }^{1}$ and Liset Menendez de la Prida ${ }^{1 \star}$ \\ ${ }^{1}$ Instituto Cajal, Consejo Superior de Investigaciones Científicas, Madrid 28002, Spain, ${ }^{2}$ Neurobiología-Investigación, Hospital Ramón y Cajal, Madrid \\ 28034, Spain, and ${ }^{3}$ Hospital Nacional de Parapléjicos, Servicio de Salud de Castilla la Mancha, Toledo 45071, Spain
}

Fast ripples are a type of transient high-frequency oscillations recorded from the epileptogenic regions of the hippocampus and the temporal cortex of epileptic humans and rodents. These events presumably reflect hypersynchronous bursting of pyramidal cells. However, the oscillatory spectral content of fast ripples varies from 250 to $800 \mathrm{~Hz}$, well above the maximal firing frequency of most hippocampal pyramidal neurons. How such high-frequency oscillations are generated is therefore unclear. Here, we combine computational simulations of fast ripples with multisite and juxtacellular recordings in vivo to examine the underlying mechanisms in the hippocampus of epileptic rats. We show that populations of bursting cells firing individually at $100-400 \mathrm{~Hz}$ can create fast ripples according to two main firing regimes: (1) in-phase synchronous firing resulting in "pure" fast ripples characterized by single spectral peaks that reflect single-cell behavior and (2) out-of-phase firing that results in "emergent" fast ripples. Using simulations, we found that fast ripples generated under these two different regimes can be quantitatively separated by their spectral characteristics, and we took advantage of this separability to examine their dynamics in vivo. We found that in-phase firing can reach frequencies up to $300 \mathrm{~Hz}$ in the $\mathrm{CA} 1$ and up to $400 \mathrm{~Hz}$ in the dentate gyrus. The organization of out-of-phase firing is determined by firing delays between cells discharging at low frequencies. The two firing regimes compete dynamically, alternating randomly from one fast ripple event to the next, and they reflect the functional dynamic organization of the different regions of the hippocampus.

\section{Introduction}

High-frequency oscillations $>200 \mathrm{~Hz}$ are commonly observed during normal and pathological activity of different brain regions, including cortical evoked potentials (Gobbelé et al., 1998; Jones and Barth, 1999), cerebellar oscillations (Adrian, 1935; Cheron et al., 2005), and subthalamic activity in Parkinson's disease (Foffani et al., 2003; López-Azcárate et al., 2010). One particular type of pathological transient high-frequency oscillation, termed fast ripples $(250-800 \mathrm{~Hz})$, is observed in local field potentials (LFPs) recorded from the hippocampus and temporal cortex of epileptic humans and rodents (Bragin et al., 1999; Urrestarazu et al., 2007; Worrell et al., 2008). Fast ripples sometimes precede epileptic seizures (Khosravani et al., 2005; Jiruska et al., 2010) and are found most reliably in epileptogenic regions (Bragin et al., 2000; Staba et al., 2007; Worrell et al., 2008). Thus,

Received June 29, 2010; revised Sept. 17, 2010; accepted 0ct. 14, 2010.

This work was supported by grants from the Spanish Ministry Ministerio de Ciencia e Innovación (MICINN) (BFU2006-10584 and BFU2009-07989), the ERANET-Neuron project EPINet cofunded by the MICINN and the European Union Commission under FP7 (EUI2009-04093) and La Fundación para la Investigación Sanitaria en Castilla la Mancha (Pl-2006/49). We thank Aric Agmon for useful comments and discussion and Richard Miles and Jozsef Csicsvari for critical comments on the manuscript. We also thank Albert Compte for inspiring discussions on the spectral entropy and Juan Aguilar for useful tips for juxtacellular recordings in vivo. L.M.P., G.F., and J.M.I. conceived the study.J.M.I. performed computational simulations; G.F. developed analytical tools and analyzed part of the data; E.C. was responsible for histological analysis and immunostaining; M.I. developed and handled the epileptic rats; and L.M.P. coordinated the study, performed the experiments, analyzed data, and wrote the paper.

*J.M.I., G.F., and L.M.P. contributed equally to this work.

Correspondence should be addressed to Dr. Liset Menendez de la Prida, Instituto Cajal, Consejo Superior de Investigaciones Científicas, Avenida Doctor Arce, 37, Madrid 28002, Spain. E-mail: Imprida@cajal.csic.es.

DOI:10.1523/JNEUROSCI.3357-10.2010

Copyright $\odot 2010$ the authors $\quad 0270-6474 / 10 / 3016249-13 \$ 15.00 / 0$ fast ripples potentially have diagnostic or predictive value, and their further study is warranted.

A decade after their discovery, the nature and mechanisms of fast ripples still remain actively investigated. The main hypothesis is that fast ripples represent a "pure" (i.e., single frequency band) oscillation reflecting population spikes of synchronously bursting neurons (Bragin et al., 2000; Dzhala and Staley, 2004). Recently, we showed that in vitro oscillations in the frequency range of fast ripples can emerge in the epileptic hippocampus from neurons bursting at lower frequencies (100-200 Hz) (Foffani et al., 2007). Bursting cells fired at half the frequency of fast ripples but in antiphase, creating a harmonic frequency-doubling effect (Staley, 2007). This effect was associated with a reduced spiketiming reliability, which decreased neuronal synchronization. We thus proposed the alternative hypothesis that fast ripples could represent emergent oscillations that arise from desynchronization, rather than hypersynchronization, of bursting cells firing at lower frequencies.

However, there are some apparent paradoxes with the harmonic hypothesis (Beenhakker and Huguenard, 2009). First, it is unclear how reduced spike-timing reliability, which presumably will tend to randomize firing times, can organize the antiphase firing that is necessary for the emergence of harmonic oscillations. Second, previous studies showed that the appearance of fast ripples correlated with precise neuronal bursting (Dzhala and Staley, 2004), which seems to contradict the proposed role of reduced spike-timing reliability. Finally, fast ripples in vivo are not always consistent with harmonic oscillations emerging from antiphase firing (Engel et al., 2009), including reports of large- 
amplitude pure oscillations up to $500 \mathrm{~Hz}$ without harmonics (Staba et al., 2002).

In this study, we first used computational simulations of pathological population discharges to derive the spectral organization of fast ripples resulting from in-phase and out-of-phase neuronal firing. We show that populations of bursting cells can create fast ripples according to two main firing regimes: pure oscillations resulting from individual in-phase firing, and emergent oscillations, resulting from out-of-phase firing-including the special case of antiphase firing that leads to harmonic fast ripples. We found that fast ripples resulting from these two different firing regimes can be quantitatively separated using measurements of their oscillatory spectra. We then combined juxtacellular and multisite recordings to further investigate fast ripple dynamics in the epileptic hippocampus in vivo. Our data show that fast ripples in vivo interchange between these two regimes, and they reflect the functional dynamic organization of the different regions of the epileptic hippocampus.

\section{Materials and Methods}

Computational simulations. Transient fast ripple oscillations observed in local field potential recordings are thought to represent population spikes from clusters of abnormally bursting neurons (Bragin et al., 2000). While many factors, including ephaptic interactions (Bikson et al., 2003), gap junctions (Draguhn et al., 1998; Traub et al., 1999; Middleton et al., 2008), and several forms of channelopathies (Richter et al., 2008), can be implicated in the generation of different forms of high-frequency oscillations, here we do not attempt to model these several mechanisms for the case of fast ripples. Instead, we focus on the spectral properties of fast ripples resulting from in-phase and out-of-phase neuronal firing during population discharges.

To this purpose, we obtained realistic simulations of field potential oscillations from a population of bursting cells using a well known model of pathological population discharges (Traub and Wong, 1982; Traub and Miles, 1991; Traub et al., 1991). Briefly, each neuron is composed of 19 equivalent cylinders representing the soma (1 compartment), the basal (8), and the apical (10) dendrites of pyramidal cells (Traub et al., 1991). The somatic compartment had a radius of 4.23 and $125 \mu \mathrm{m}$ length. The apical compartments were $120 \mu \mathrm{m}$ length with a radius of $2.89 \mu \mathrm{m}$, and basilar compartments were $110 \mu \mathrm{m}$ length and radius of $2.42 \mu \mathrm{m}$. This gives realistic values of somatic $\left(3320 \mu \mathrm{m}^{2}\right)$ and dendritic total surface areas $\left(35,170 \mu \mathrm{m}^{2}\right)$. Each compartment was equipped with a set of voltage-dependent ionic conductances according to the Hodgkin-Huxley formalism and a specific spatial channel distribution tuned to produce intrinsic bursting (Traub et al., 1991). Ionic currents included the following: inactivating $\mathrm{Na}^{+}$, high-threshold $\mathrm{Ca}^{2+}$, and four $\mathrm{K}^{+}$conductances (delayed rectifier, transient, or the A-type current, slow afterhyperpolarization, and the rapid voltage- and $\mathrm{Ca}^{2+}$-dependent C-type current). The reversal potentials were $E_{\mathrm{Na}}=45, E_{\mathrm{K}}=-75$, and $E_{\mathrm{Ca}}=80 \mathrm{mV}$. As in Traub et al. (1991), we also simulated a pool of intracellular calcium at each compartment following a first-order kinetic equation.

The membrane capacitance $\left(C_{\mathrm{m}}\right)$ of all compartments was 3 $\mu \mathrm{F} \cdot \mathrm{cm}^{-2}$, the internal resistivity $\left(R_{\mathrm{i}}\right) 100 \Omega \cdot \mathrm{cm}$, and the membrane resistivity $\left(R_{\mathrm{m}}\right) 10,000 \Omega \cdot \mathrm{cm}^{2}$. To obtain a wider range of intraburst frequencies from 180 to $500 \mathrm{~Hz}$, the kinetics of the $\mathrm{Na}^{+}$channel was accelerated (by dividing its activation time constant by a factor of 2 ) and the activation and inactivation curves of $\mathrm{Na}^{+}$channels were shifted $5 \mathrm{mV}$ in the depolarization direction. The densities of $\mathrm{Ca}^{2+}, \mathrm{Na}^{+}$, and $\mathrm{K}^{+}$ channel conductances were varied slightly within the typically used ranges (Traub et al., 1991). We also lowered $C_{\mathrm{m}}$ to $1 \mu \mathrm{F} \cdot \mathrm{cm}^{-2}$ to obtain intraburst frequencies between 200 and $400 \mathrm{~Hz}$ and up to $0.8 \mu \mathrm{F} \cdot \mathrm{cm}^{-2}$ to reach firing rates faster than $400 \mathrm{~Hz}$. All these parameter values are within the standards used in computational modeling (Traub et al., 1989, 1991; Traub and Miles, 1991).

The network consisted of 120 neurons with their somata aligned to form a cellular stratum of four neuronal layers and the dendritic axis oriented orthogonally, expanding in a spiral-like distribution from the center. The spiral step radius was $12 \mu \mathrm{m}$ so that the whole population occupied a cylinder of $100 \mu \mathrm{m}$ radius. We also simulated a network of 1000 neurons, which occupied a cylinder of $\sim 300 \mu \mathrm{m}$ radius. To look at the effect of out-of-phase firing, we simulated two clusters of 60 neurons that burst at different delays. For simulations of the two clusters, cells belonging to each cluster alternate position in the spiral. The network architecture was random with a connection probability of $p=0.02-0.03$ (Traub and Miles, 1991). Fast excitatory AMPA-like synaptic conductances were simulated using the exponential $\alpha$ function:

$$
g(t)=g_{\text {syn }} \cdot \frac{t}{\tau_{\text {syn }}} \cdot e^{1-\frac{t}{\tau_{\text {sn }}}},
$$

with $\tau_{\text {syn }}$ of 2 ms and $g_{\text {syn }}$ being the maximal strength of the individual synapses, which was varied from 4 to $28 \mathrm{nS}$ in different simulations. The reversal potential of glutamatergic currents was set at $0 \mathrm{mV}$. Synapses were modeled to occur at the apical compartment at $120 \mu \mathrm{m}$ from the soma. Synaptic noise consisted of excitatory ( $g_{\text {syn }}$, varied from 0.4 to 2.2 $\mathrm{nS}$ ) and fast inhibitory $\left(g_{\text {syn }}=0.75 \mathrm{nS}\right)$ conductances being injected at the apical compartment (at $240 \mu \mathrm{m}$ from the soma) at random timing with a rate of $50 \mathrm{~Hz}$ for excitation and $60 \mathrm{~Hz}$ for inhibition. The reversal potential for $\mathrm{GABA}_{\mathrm{A}}$-like synaptic currents was $-75 \mathrm{mV}$.

Hodgkin-Huxley simulations were performed using the GENESIS platform (http://genesis-sim.org/) on a PC computer running under SuSE Linux v.9. At the simulation onset, one cell was made to burst, and the activity spontaneously spread in the population. One population event was generated per each simulation, representing $200 \mathrm{~ms}$ of real time. An exponential Euler explicit method was used with integration time steps of $5 \mu \mathrm{s}$.

To obtain realistic extracellular field potentials, we applied a coordinate transformation to the equivalent cylinders that matches real neuronal distances. Thus, total currents obtained from the 19 compartments were considered as being produced by 19 nodes distributed alongside the typical basal-to-apical length of hippocampal neurons $(800 \mu \mathrm{m})$. The extracellular potentials were calculated by means of the field equation (Malmivuo and Plonsey, 1995):

$$
\Phi(r, t)=\frac{1}{4 \pi \sigma} \cdot \sum_{i=1}^{n} \frac{I_{i}(t)}{r_{i}},
$$

where $\Phi$ is the field potential, $I_{i}(t)$ is the total current flowing from the $i$ th node into the brain tissue of conductivity $\sigma$, and $r_{i}$ is the distance of the $i$ th node to the recording site $r$. We assumed that the extracellular medium was isotropic with a mean conductivity of $300 \Omega \cdot \mathrm{cm}$ (Varona et al., 2000). Field potentials were calculated at the cellular stratum at the center of the population.

Generation of epileptic rats. Rats were rendered chronically epileptic using a single unilateral intrahippocampal injection of kainic acid, as previously described (Bragin et al., 1999). Adult Wistar rats (180-200 g) were anesthetized with $1.5-2 \%$ isoflurane mixed with oxygen (400-800 $\mathrm{ml} / \mathrm{min}$ ), fixed to a stereotaxic apparatus, and unilaterally injected with $0.8 \mu \mathrm{l}$ of kainic acid $(0.4 \mathrm{mg} / 0.2 \mathrm{ml}$ in saline $)$ in the right hippocampus (AP: 4.4, ML: 4.0, DV: 3.4). The surgery was terminated after $10 \mathrm{~min}$ of injection, and the rat was then disconnected from the anesthesia and observed for signs of status epilepticus. Diazepam ( $4 \mathrm{mg} / \mathrm{kg}$, i.p.) was injected $1 \mathrm{~h}$ after the status and repeated if needed. Animals were systemically injected with $2.5 \mathrm{ml}$ of $5 \%$ dextrose several times per day, and diet was supplemented with fruit and powder milk during the following 2-3 d.

Rats were observed for behavioral and clinical signs of spontaneous seizures at random times between 8:00 A.M. and 7:00 P.M. during at least 20 min and 3-4 times per week. Seizures were scored according to Racine (1972). Spontaneous tonic-clonic seizures consisting of forelimb automatisms were observed in 12 of 14 rats after $2-5$ weeks postinjection. In two rats, only orofacial automatisms and body rigidity were observed, consistent with low Racine scores. These animals were not excluded from the database since no obvious differences were found in their fast ripple recordings compared to the other rats. In all 14 rats, the middle and 
caudal part of the injected hippocampus was severely disrupted with massive cell loss in all regions. The dorsal tip exhibited prominent cell loss in the CA3 region and the hilus but a relatively preserved CA1 region and dentate granule cell layer, whereas the contralateral hippocampus was normal (supplemental Fig. $1 A, B$, available at www.jneurosci.org as supplemental material). All procedures concerning animals met the European guidelines (86/609/EEC).

In vivo electrophysiology. Epileptic rats were anesthetized with urethane (1.5-2.0 g/kg, i.p.) and fastened to the stereotaxic frame. Body temperature was kept constant at $37^{\circ} \mathrm{C}$ with a heating blanket. Small holes of $1.5 \mathrm{~mm}$ diameter were drilled above the hippocampus contralateral to the kainic acid injection for recordings (AP: -4.4, ML: 3.5) and stimulation (AP: -1.2 , ML: 2.9). A craniotomy of $\sim 5.8 \mathrm{~mm}$ anteroposterior and $4 \mathrm{~mm}$ mediolateral was performed above the hippocampus ipsilateral to the kainic acid injection to facilitate localization of regions generating fast ripples. The dura was gently removed around the targeted region to promote stability. A concentric bipolar stimulating electrode was advanced $4.0 \mathrm{~mm}$ at a $30^{\circ}$ angle to target the CA3 region contralateral to the injection. A second stimulating electrode was advanced vertically $3.0 \mathrm{~mm}$ to target the perforant pathway at the hemisphere ipsilateral to the injection (AP: $-7.0 \mathrm{~mm}$ and ML: $3.5 \mathrm{~mm}$ ). Stimulation consisted of biphasic square pulses of $0.2 \mathrm{~ms}$ duration and amplitudes of $0.1-1 \mathrm{~mA}$ every $15 \mathrm{~s}$. A subcutaneous $\mathrm{Ag} / \mathrm{AgCl}$ wire was placed in the neck as a reference electrode.

Multisite recordings were obtained with linear silicon probes of 16 electrodes at $100 \mu \mathrm{m}$ vertical spacing (NeuroNexus Tech). They were positioned to record from all strata simultaneously, from the CA1 to the dentate gyrus. Extracellular signals were preamplified $(4 \times$ gain $)$ and recorded with a 16-channel AC amplifier (Multichannel Systems), further amplified by 100 , filtered by analog means at $1 \mathrm{~Hz}$ to $5 \mathrm{kHz}$, and sampled at $20 \mathrm{kHz} /$ channel with 12 bit precision. Data were exported to MATLAB for further analysis.

Silicon probes were positioned guided by extracellular stimulation. At the hippocampus contralateral to the kainic acid injection, the CA1 region was identified by its characteristic population spike response to suprathreshold CA3 stimulation (supplemental Fig. 1C, available at www. jneurosci.org as supplemental material). Once the position of the CA3 stimulating electrode was optimized at the contralateral hippocampus, we moved the 16-channel probe to the side ipsilateral to injection to look for regions generating fast ripples. They were detected by their characteristic stimulation-evoked responses consisting of population discharges, in contrast to the normal single population spike recorded in the contralateral side (supplemental Fig. $1 D$, available at www.jneurosci.org as supplemental material). Spontaneous fast ripples occurred sporadically at the same regions in the hippocampus ipsilateral to the injection. In some cases, we noted that these spontaneous events appeared after 2-4 stimulation pulses of the contralateral CA3 at 1-1.5 mA. Several tracks needed to be tested to localize regions generating fast ripples, with optimal coordinates typically found between $-2.9 \mathrm{~mm}$ and -3.7 $\mathrm{mm}$ anteroposterior and from 1.5 to $2.9 \mathrm{~mm}$ mediolateral, variable from rat to rat. One-dimensional current source density (CSD) profiles were calculated using the second spatial derivate of local field potentials. Smoothing was not applied and no resistivity difference between strata was considered. After completing recordings, the silicon probe was carefully withdrawn in the ventrodorsal direction without changing other coordinates. The probe was then submerged in a solution containing the red fluorescent dye $1,1^{\prime}$-dioctadecyl$3,3,3^{\prime}, 3^{\prime}$-tetramethylindocarbocyanine perchlorate (DiI) (Invitrogen) while still in the stereotaxic frame and reinserted for subsequent histological localization. Electrophysiological signals before and after DiI staining were similar.

Juxtacellular recordings were obtained blindly, using an intracellular amplifier (Axoclamp 2B), guided by extracellular stimulation. We used glass pipettes of $15-25 \mathrm{M} \Omega$ filled with $0.5 \mathrm{M} \mathrm{NaCl}$ driven by a hydraulic manipulator (Narishige). Units were recorded at the stratum pyramidale of the CA1 region and at the granule cell layer of the dentate gyrus, as assessed by online comparison of field potential responses between the glass electrode and the corresponding contact in the silicon probe. We monitored the shape and amplitude of spikes over the course of experiments, to check for firing pattern stability.

Histology and immunohistochemistry. After completing in vivo experiments, rats were perfused intracardially with $100 \mathrm{ml}$ of PBS and $0.2 \%$ heparin followed by $400 \mathrm{ml}$ of $4 \%$ paraformaldehyde in PBS, pH 7.3. The brains were then removed and stored in the fixative solution overnight. Coronal sections of $100 \mu \mathrm{m}$ were cut with a vibratome. For immunostaining, free-floating sections were washed in $0.1 \mathrm{~m}$ PBS followed by incubation in $1 \% \mathrm{H}_{2} \mathrm{O}_{2}$ for $45 \mathrm{~min}$. After washing in PBS several times, sections were maintained during $1 \mathrm{~h}$ in PBS containing $10 \%$ fetal bovine serum (FBS) and $0.25 \%$ Triton and then incubated overnight at $4^{\circ} \mathrm{C}$ in antisera to NeuN (in mouse, 1:1000, Bachem) diluted in PBS containing $1 \%$ FBS and $0.25 \%$ Triton. On the second day, sections were incubated for $2 \mathrm{~h}$ in biotinylated anti-mouse (1:200) and for $1 \mathrm{~h}$ in avidin-biotinhorseradish peroxidase complex (1:1000, Vector Laboratories) diluted in PBS. They were washed in PBS, developed in $0.05 \%$ diaminobenzidine tetrahydrochloride and $0.01 \% \mathrm{H}_{2} \mathrm{O}_{2}$, and mounted on slides coverslipped with Eukitt (Fluka). In some cases, we used the Alexa-568 antimouse antibody (Invitrogen) and directly mounted the sections in $87 \%$ glycerol. The DiI staining was directly observed in the sections.

Data analysis. In our previous work, we studied the spectral properties of fast ripples in vitro averaged over many events using standard fast Fourier transform (FFT) techniques (Foffani et al., 2007). In the present study, we needed to quantify the dynamics of fast ripples in vivo on a single-event basis. Single-event power spectra of field potential recordings were thus estimated with a more sophisticated approach based on the Thomson multitaper method (Thomson, 1982). We specifically adopted the indirect method, estimating the spectrum from the autocorrelation function of the signal, after mean detrending and with a timebandwidth product of 2 (i.e., 3 tapers). Epochs of $200 \mathrm{~ms}$ of fast ripple events were detected by eye and triggered by the positive peak of the accompanying individual sharp waves. We used FFT sizes to obtain frequency resolutions between 4.9 and $19.5 \mathrm{~Hz}$. Time-frequency single-event representations were obtained by applying the multitaper spectral estimation in sliding windows with $97.7 \%$ overlap and a frequency resolution of $10 \mathrm{~Hz}$. Note that the time-frequency resolution was optimized to quantify the overall single-event spectrum and not to quantify the spectral dynamics within events (Nguyen et al., 2009).

To quantify the spectral organization, each single-event spectrum was normalized by the power between 100 and $800 \mathrm{~Hz}$. The normalized power was treated as a statistical distribution, from which we extracted three main parameters: the entropy, a fast ripple index (FR index), and the spectral mode. The entropy, which is a measure of spectral disorganization, was calculated as $-\sum_{f} p(f) \log _{2}(p(f))$, where $p(f)$ is the normalized power at each frequency $f$. By definition, the entropy does not depend on the frequency and is maximal for a flat spectrum (noisy oscillations) at $\log _{2} N=5.12$, with $N=35$ being the number of bins used. The FR index was calculated as the integral of the normalized power in the $400-800 \mathrm{~Hz}$ band, and represents the proportion of the spectrum at the upper fast ripple band. It varies from 0 to 1 . Finally, the spectral mode represents the most probable frequency peak of the spectrum. Field potentials from simulations and in vivo experiments were all analyzed in the same way. In all cases, signals were filtered using forwardbackward zero-phase FIR filters of order 512.

Action potentials from simulations and from juxtacellular recordings were discriminated using standard threshold-based procedures on highpass filtered data and checked visually.

In simulations, we calculated a pairwise synchronization measurement of the burst synchrony between neurons using the interspike interval (ISI) distance (Kreuz et al., 2009). For this purpose, we used the instantaneous coefficient of variation of the ISI taken across all neuron pairs at any given time over the entire temporal window. Values at zero indicate perfect synchronization between pairs, whereas the larger the coefficient of variation, the poorer the firing coordination. Results are reported in a matrix form for all pairwise combinations.

In juxtacellular recordings, the firing frequency of units during individual fast ripple events, as detected in the adjacent field potential recording, was estimated as the inverse of the average ISI for bursts of more than two spikes. The firing precision of units within the fast ripple cycles was 
estimated by calculating the corresponding Hilbert transform of the filtered $(100-800 \mathrm{~Hz})$ nearby field potential. We then extracted the phase of the fast ripple oscillation in correspondence to every spike of the unit, considering only spikes occurring when the amplitude of fast ripples was at least half of the maximal amplitude within an event. We calculated the firing precision as the phase jitter of the spikes, converted from radians to milliseconds through the single-event spectral mode of the nearby field potential.

All results are given as mean \pm SD. Correlations were assessed using Pearson's correlation coefficient.

\section{Results}

Fast ripples are transient high-frequency oscillation recorded in the epileptic hippocampus that are thought to reflect population discharges from abnormally bursting neurons (Fig. 1A) (Bragin et al., 2000; Dzhala and Staley, 2004). Here we used a widely used computational model of pathological population discharges to simulate field potential fast ripple oscillations associated with neuronal bursting (Traub and Wong, 1982; Traub and Miles, 1991; Traub et al., 1991). Our aim was to derive predictions on the spectral organization of local field potential oscillations depending on in-phase and out-of-phase neuronal firing at different frequencies and to test these predictions in vivo.

\section{Computational simulations of population fast ripple oscillations}

We first sought to simulate fast ripple oscillations associated with population discharges by using a computational model of pathological hippocampal networks (see Traub and Miles, 1991, for a summary). For this purpose, we built a network of 120 intrinsically bursting neurons having 19 compartments that were equipped with voltage-dependent ionic conductances (see Materials and Methods). Neurons were connected randomly through fast excitatory AMPA-like synapses, and extracellular potentials were calculated using the field equation (Malmivuo and Plonsey, 1995) (see Materials and Methods).

At the onset of the simulation, one neuron fired a burst consisting of a group of six action potentials at a frequency of $180 \mathrm{~Hz}$. The firing of this single neuron initiated a rapid buildup of activity that spread across excitatory synapses and led to bursting in the entire population (Fig. $1 \mathrm{~B}$ ), as described both experimentally and computationally (Traub and Wong, 1982; Miles and Wong, 1983; Menendez de la Prida et al., 2006). Simulated field potential recordings at the cell body layer showed population events with high-frequency oscillations (Fig. $1 B$ ). Most cells fired within tens of milliseconds of the triggering burst and contributed to generate the field potential oscillations (Fig. $1 B$, black trace). Some cells remained hyperpolarized after an initial burst, revealing part of the underlying compound population EPSP (Fig. $1 \mathrm{~B}$, blue
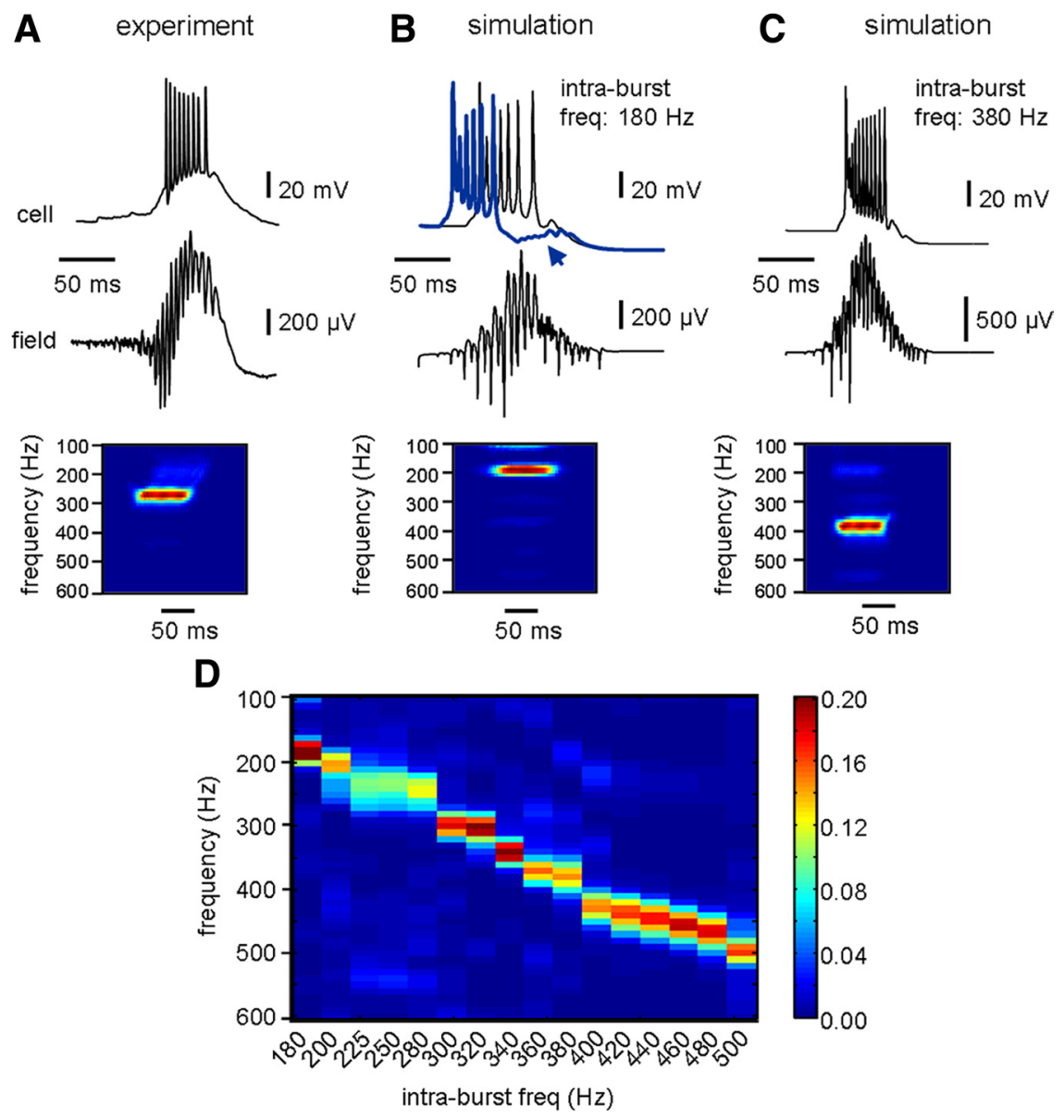

Figure 1. Computational simulations of fast ripple oscillations. $A$, Fast ripples are thought to reflect population spikes from synchronous bursting cells. Here is an example of one spontaneous population discharge and the associated fast ripples as recorded

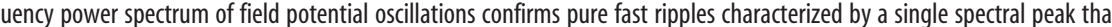
connected intrinsically bursting neurons. At the onset of the simulation, one neuron fires a burst and recruits other neurons to fire. Most cells fire after tens of milliseconds of the triggering burst and contribute to generate field potential oscillations (black trace). Some cells remain hyperpolarized after an initial burst and reveal part of the underlying population EPSP (blue trace, arrow). Fast ipple oscillations at $180 \mathrm{~Hz}$ are detected at the field potential, which coincides with the intraburst frequency of single cells. $C$

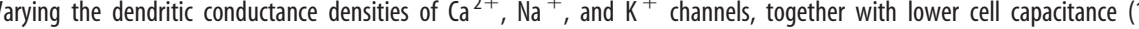
$\left.\mu \mathrm{F} \cdot \mathrm{cm}^{-2}\right)$, accelerated the intraburst frequency of individual neurons. As a result, high-frequency oscillations resulting at the field potential shifted in a similar frequency range. Here is shown the case of an intraburst frequency of $380 \mathrm{~Hz}$. D, Spectral peaks of pure single frequency field potential oscillations resulting from simulations with different intraburst frequencies.

trace, arrow). These features are consistent with previous models and experiments examining the mechanisms of pathological population discharges (see Traub and Miles, 1991 for a summary). Here, we will focus on the emergence of the accompanying high-frequency field potential oscillations.

\section{Spectral organization of high-frequency oscillations in the ripple and fast ripple bands}

In our basic simulation, the spectrum of fast ripples exhibited a single peak at $\sim 180 \mathrm{~Hz}$, which reflected the intraburst frequency of single neurons (Fig. $1 B$ ). Varying slightly the dendritic conductances of $\mathrm{Ca}^{2+}, \mathrm{Na}^{+}$, and $\mathrm{K}^{+}$channels within the ranges typically used in the modeling literature, together with a lower cell capacitance $\left(0.8-1 \mu \mathrm{F} \cdot \mathrm{cm}^{-2}\right)$ and a faster $\mathrm{Na}^{+}$dynamics, we were able to modulate the frequency of single-cell bursting between 180 and $500 \mathrm{~Hz}$. We then repeated the simulation for each intraburst frequency and looked at the field potential oscil- 
A1

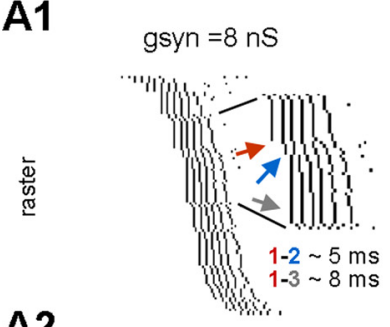

A2

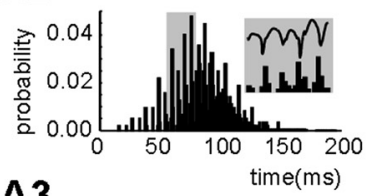

A3

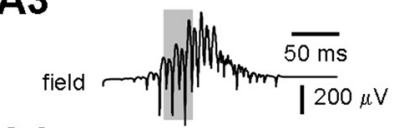

A4

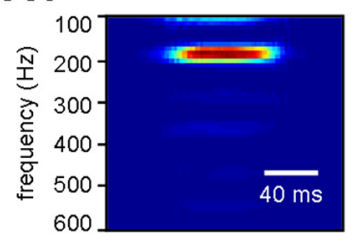

D

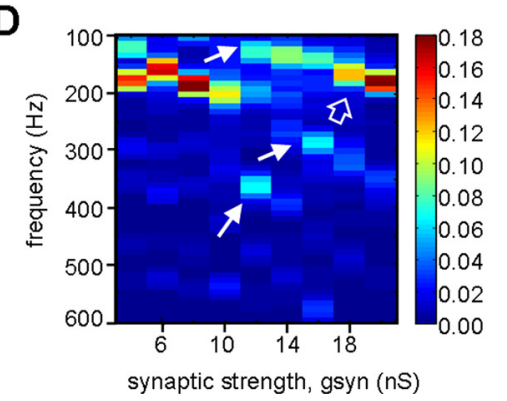

B1

B3

\section{B4}
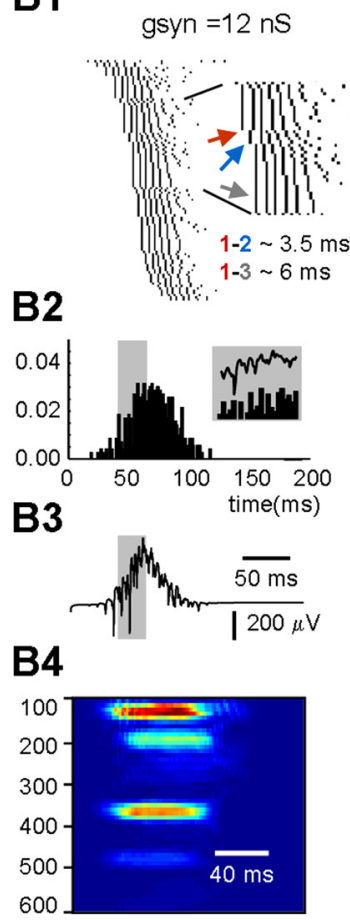

E

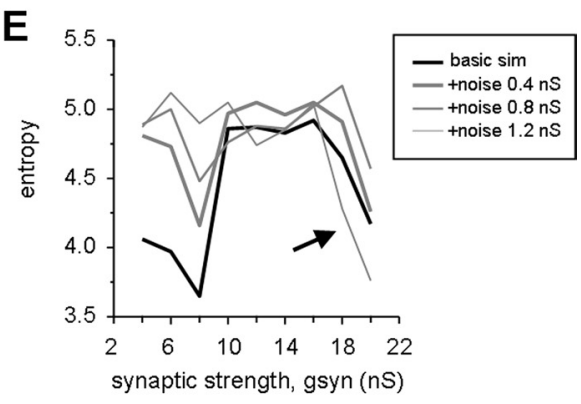

Figure 2. Spectral features of emergent high-frequency field potential oscillations. A raster representation of the firing from all neurons in the simulation facilitates the interpretation of how field potential oscillations develop. $A-C$, Results are illustrated in a columnar arrangement for simulations using two different synaptic strengths: $g_{\text {syn }}=8 \mathrm{nS}(\boldsymbol{A})$ and $g_{\text {syn }}=12 \mathrm{nS}(\boldsymbol{B})$; and for $g_{\text {syn }}=8 \mathrm{nS}$ plus added glutamatergic $(0.8 \mathrm{nS})$ and GABAergic $(0.75 \mathrm{nS})$ synaptic noise $(\boldsymbol{C})$. In all cases, the intraburst frequency of individual neuronal bursting was $180 \mathrm{~Hz} . \mathbf{A 1}, \mathbf{B 1}, \mathrm{C1}$, Rasters are ordered according to the timing of individual neuronal firing in the simulation shown in $A 1$. Three neuronal clusters are indicated with red, blue, and gray arrows to emphasize their activation delays and enlarged at right. $\mathbf{A} \mathbf{2}, \mathbf{B 2}, \mathbf{C}$, Firing histograms obtained from the rasters shown above. The insets show histogram details indicated in gray, together with the concurrent field potential oscillation. $A 3, B 3, C 3$, Simulated field potential recordings show population events with fast ripple oscillations. $A 4, B 4, C 4$, Time-frequency analysis of the fast ripple events shown above. Power spectrum values are color coded from zero to maximum. $\boldsymbol{D}$, Spectral peaks of fast ripple oscillations resulting from simulations with different synaptic strength. Note the emergence of frequency components faster and slower than $180 \mathrm{~Hz}$ for $g_{\text {syn }}>10 \mathrm{nS}$ (white arrows). For synaptic strength $g_{\text {syn }} \geq 18 \mathrm{nS}$, pure single-peaked oscillations are reinstated (open arrow). $\boldsymbol{E}$, Plot of the spectral entropy, which is a measure of the spectral disorganization, against the synaptic strength for the simulations shown in $\boldsymbol{D}$ (black trace) and for three levels of glutamatergic synaptic noise (gray traces).

lations. For increasing intraburst frequencies, field potential oscillations shifted in a similar range, from the ripple $(100-200 \mathrm{~Hz})$ to the fast ripple $(>250 \mathrm{~Hz}$ ) band (Fig. 1C,D). In all these cases, fast ripples were brought about by synchronous neuronal bursting at the intrinsic frequency of individual neurons. We termed these oscillations "pure fast ripples," because their spectrum was composed of a single peak that reflected single-cell behavior.

An ordered raster representation of the firing from all neurons of the simulation illustrates the single-cell behavior (Fig. 2A1). The firing histogram shows that action potentials from different neurons occurring within $1 \mathrm{~ms}$ bin contributed together to form a population spike (Fig. $2 \mathrm{~A} 2$, inset). Neurons typically organized

in clusters, which were determined by both the propagation delays and the intrinsic intraburst frequency. Propagation delays depended on the temporal summation required to bring postsynaptic cells to the firing threshold. In Figure 2A1, cells in the cluster marked by a red arrow were recruited to burst together $5 \mathrm{~ms}$ before cells in the cluster marked by a blue arrow, and $\sim 8 \mathrm{~ms}$ before cells in the cluster marked in gray. Population spikes were contributed by individual action potentials from different clusters due to overlap between propagation delays and intraburst interspike intervals. For instance, first spikes in cluster 3 (gray arrow) became synchronous with third spikes in cluster 1 (red arrow), thus contributing together to the same ripple cycle (Fig. $2 A 1)$. This cluster organization is captured by a measure of pairwise spike synchrony (Kreuz et al., 2009) (supplemental Fig. $2 A$, available at www.jneurosci.org as supplemental material; see Materials and Methods).

Increasing the strength of the individual synapses had a major effect: it accelerated polysynaptic propagation by bringing neurons earlier to firing threshold, while maintaining a similar intraburst frequency (Fig. 2B1). This introduced a temporal mismatch between propagation delays and intraburst interspike interval, which resulted in out-of phase firing (see delays between clusters 1, 2, and 3 in Fig. $2 B 1$ vs Fig. 2A1). As a consequence, neuronal synchronization decreased and cluster composition broke down into subclusters (supplemental Fig. $2 B$, available at www.jneurosci.org as supplemental material). This created additional population spikes (Fig. 2 B2, inset) and high-frequency components in the spectrum (Fig. 2B3,B4). The spectral distribution varied as synaptic strength increased (Fig. 2D), but note that not all the emergent peaks were strictly harmonics (Fig. 2D, white arrows). Increasing the synaptic strength resulted in increased values of the spectral entropy (Foffani et al., 2007), which is a measure of the spectral disorganization (Fig. 2E, thick black line). In this regime of increased synaptic strength, fast ripples are not pure oscillations reflecting single-cell behavior, but rather become emergent oscillations that can reach frequency values much higher than the firing frequency of individual cells. Further increasing the synaptic strength ( $\geq 18 \mathrm{nS})$ reorganized the clusters in-phase by aligning propagation delays with the intraburst interspike interval (Fig. $2 D$, open arrow). Hence, pure oscillations were reinstated at the cell intrinsic frequency and the spectral entropy decreased (Fig. 2E, black traces). Similar features were present in simulations of 1000 bursting neurons with intraburst frequency of $180 \mathrm{~Hz}$ (supplemental Fig. 3, available at www. jneurosci.org as supplemental material). 
A

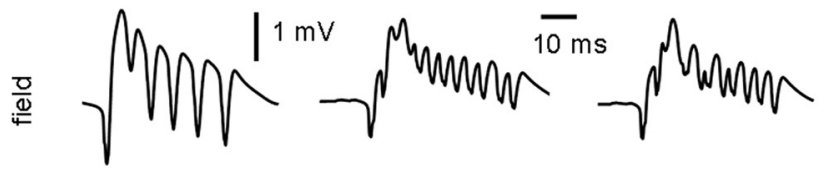
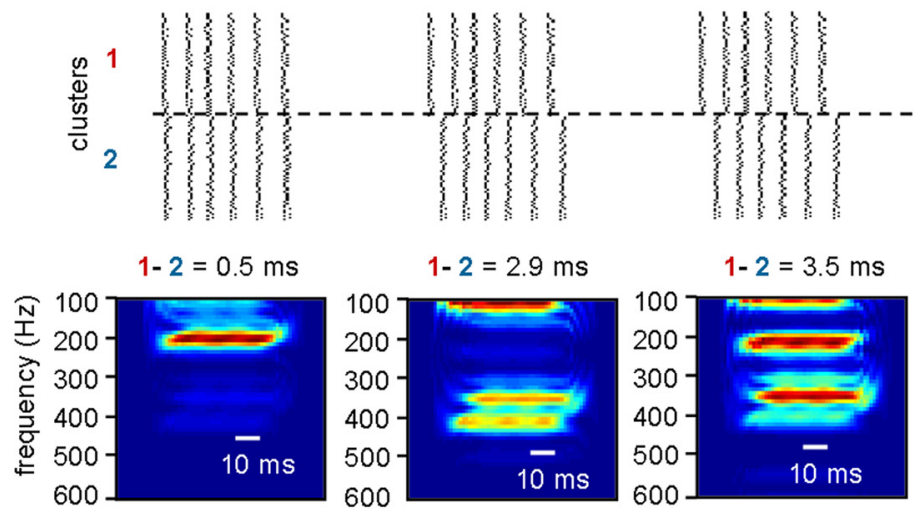

C
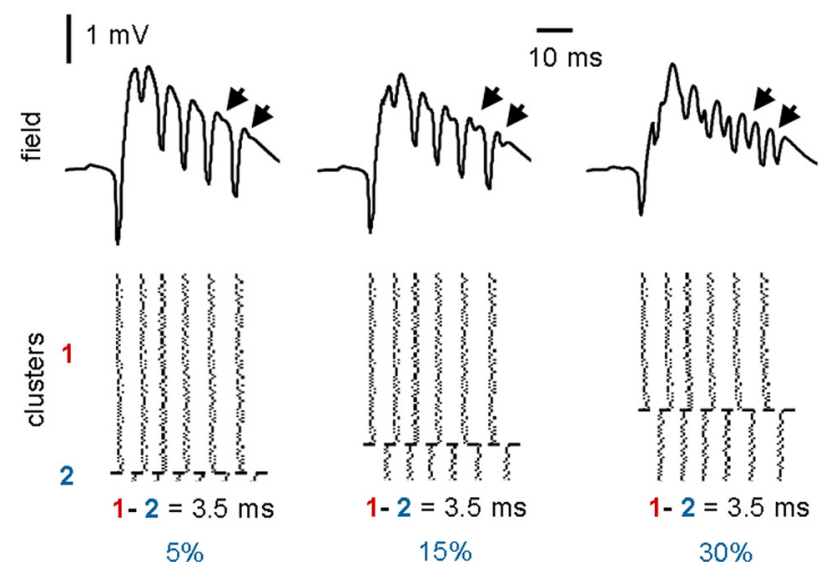

$15 \%$
B

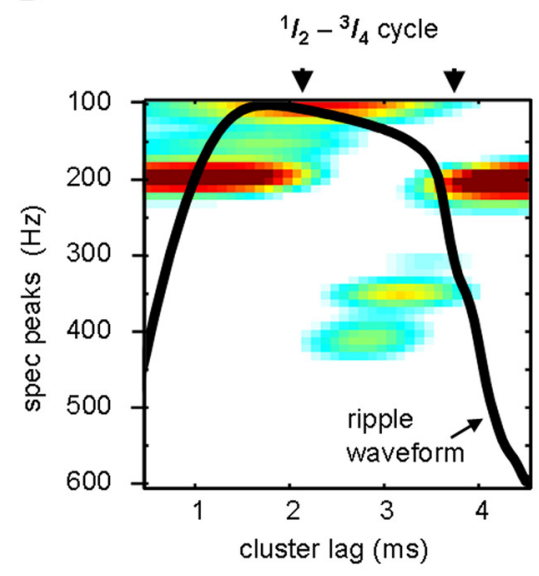

D

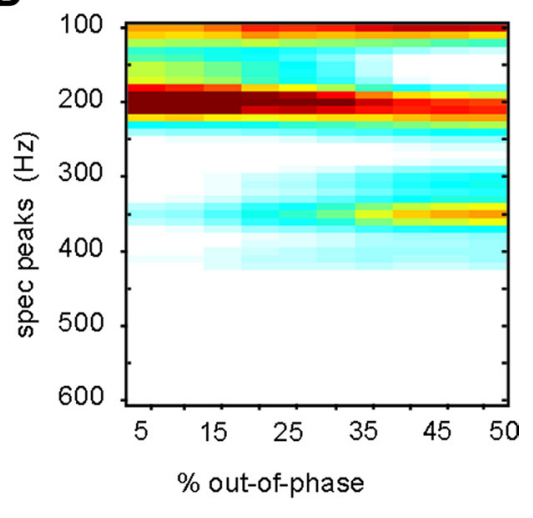

Figure 3. Temporal features of emergent fast ripple oscillations. $\boldsymbol{A}$, Simulated field potentials and rasters generated by two nonconnected clusters that fire independently at an intraburst frequency of $180 \mathrm{~Hz}$ with lags of $0.5 \mathrm{~ms}$ (left), $2.9 \mathrm{~ms}$ (middle), and $3.5 \mathrm{~ms}$ (right). Note the emergent peaks in the time-frequency spectrum for 2.9 and $3.5 \mathrm{~ms}$ lags. $\boldsymbol{B}$, Dependence of the spectral peaks on the cluster lag, which was changed in the range of 0.5 to $4.5 \mathrm{~ms}$ at $0.1 \mathrm{~ms}$ steps. Note the emergence of spectral components of the basic oscillation at $180 \mathrm{~Hz}$. A ripple waveform was scaled on the cluster lag axis to highlight the relationship between lags and the oscillatory cycle. $C$, Simulated field potentials and rasters generated by clusters of different sizes that fire independently at an intraburst frequency of $180 \mathrm{~Hz}$ with a lag of $3.5 \mathrm{~ms}$. The percentage values indicate the proportion of the whole population (clusters $1+2$ ) that fire out-of-phase (cluster 2). Arrows point to the out-of-phase cycles contributed by cells in cluster 2. D, Dependence of the spectral peaks on the percentage of neurons firing out-of-phase.

We also tested the effect of synaptic noise, as we previously found that it was related to the emergence of fast ripples in vitro (Foffani et al., 2007). Synaptic noise had nontrivial effects on network dynamics: first, it reduced spike-timing reliability at the single-cell level (Fig. 2C1), as previously described (Foffani et al., 2007). Second, it interfered with propagation delays by advancing and delaying the onset of bursting in different neurons. This produced a disorganization of bursting both within and between cells, which resulted in further subclustering of the original groups and created emergent oscillations at higher frequencies (Fig. $2 C 3, C 4$ vs Fig. $2 A 3, A 4$; supplemental Fig. $2 C$, available at www.jneurosci.org as supplemental material). Consistently, increasing the noise level at different synaptic strengths further disorganized the power spectrum, as captured by the spectral entropy (Fig. 2E, gray lines). Interestingly, there are some noise regimes for which clusters realigned and the power spectrum was reorganized, showing low entropy values (Fig. $2 E$, arrow).

In summary, these simulations suggest that fast ripples can represent either pure oscillations (Fig. $1 B-D$ ) or emergent oscillations (Fig. $2 B-D$ ). The spectral features of these oscillations depend on the interaction between a fast time scale representing the intrinsic bursting dynamic and a slower dynamic underlying polysynaptic recruitment of clusters of cells. Such a clustering organizes individual neuronal firing either in-phase, producing pure oscillations due to synchronous neuronal bursting, or outof-phase, producing emergent oscillations at higher frequencies. The increased connectivity and enhanced synaptic noise, resulting from sprouting and other forms of proepileptic circuit reorganization in vivo (Nadler et al., 1980; Esclapez et al., 1999; Shao and Dudek, 2004), might therefore interfere with the in-phase organization of firing and result in emergent oscillations.

\section{Clustering delays underlying emergent oscillations}

Is there a precise delay between two clusters that is responsible for the emergent oscillations at higher frequencies? To test for conditions required for emergent oscillations, we simulated two nonconnected and homogenously distributed clusters that independently fired at $180 \mathrm{~Hz}$ with different delays. Simulated field potential recordings were obtained at the cell layer center, with similar contribution from each cluster.

Cluster delays of $0.5-1.5 \mathrm{~ms}$ had no discernible effect on the field potential, meaning that individual action potentials from both groups contributed together to the same population spike (Fig. 3A, left). This highlights the importance of firing precision 
at the millisecond scale in setting ripples formed by population spikes (Andersen et al., 1971). A virtual elimination of the spectral peak representing the firing frequency of individual neurons occurred for cluster delays of 2.2-3.4 ms (Fig. 3A, middle panel). Under this condition, high-frequency peaks were clearly present in the spectrum, but these oscillations were all emergent, because individual neurons were firing at a lower frequency that was absent in the spectrum. Note also that the emergent oscillations do not need to be strictly harmonics of the firing frequency of individual neurons. Finally, cluster delays from 3.5 to $4 \mathrm{~ms}$ resulted in different contribution of the firing frequency of individual neurons and in emergent oscillations at higher frequencies (Fig. 3A, right). The resulting picture is more obvious if we plot the spectral peaks as a function of the cluster lag (Fig. 3B). A ripple waveform was scaled on the cluster lag axis to highlight the relationship between the emergent spectral peaks and the phase of the cycle. Out-of-phase firing of individual neurons with delays equivalent to $1 / 2$ to $3 / 4$ of the cycle resulted in emergent oscillations at high frequencies (Fig. 3B).

Emergent fast ripples caused by out-of-phase firing might also depend on the proportion of cells belonging to each cluster. We tested the effect of the size of the out-of-phase group on the spectral properties of emergent oscillations. To this purpose, we varied the proportion of cells firing out-of-phase, i.e., the relative size of clusters 1 and 2, at different delays. Figure 3, $C$ and $D$, illustrates the case of a $3.5 \mathrm{~ms} \mathrm{lag}$, which resulted in the spectrum shown in Figure $3 A$ (right) for clusters of similar sizes. We found that the relative contribution of the out-of-phase cluster to the emergent high-frequency oscillations depended on the amplitude difference between population spikes contributed by each cluster (Fig. 3C, arrows). In the resulting spectra, emergent highfrequency peaks mirrored the proportional increase of cells from the lagged cluster, being maximal for clusters of similar sizes (Fig. $3 D$ ). Therefore, out-of-phase firing among clusters of neurons will always contribute to create emergent high-frequency oscillations of increasing relative power depending on the proportional size of the clusters.

\section{Spectral characterization of fast ripple dynamics}

A major question is whether experimental fast ripples in the upper frequency band $(400-800 \mathrm{~Hz})$ can represent pure oscillations due to synchronous neuronal firing or are all emergent oscillations. To shed light on this issue, we used two spectral measures to further characterize simulated fast ripples, obtaining a bidimensional representation that will be useful to characterize the fast ripple dynamic in vivo. Specifically, we used the normalized power spectrum of fast ripple oscillations to calculate the spectrum entropy, which does not depend on the frequency, and the FR index defined between 0 and 1 as the proportion of the spectral power in the upper fast ripple band. A high FR index indicates that field potential oscillations mostly contribute to the upper fast ripple band (Fig. $4 \mathrm{~A}$ ), whereas a low FR index indicates dominant oscillations slower than $400 \mathrm{~Hz}$ (Fig. 4B). Thus the FR index quantifies the amount of spectral power in the upper fast ripple band but does not inform on the pure or the emergent character of the oscillation. Pure oscillations exhibit single spectral peaks (Fig. $4 A, B$ ) and thus have lower entropy values than emergent oscillations, which typically exhibit multiple spectral peaks (Fig. $4 C)$. Thus, pure oscillations at the upper fast ripple band will have low values of entropy together with high values of the FR index. Hence, both indexes together inform on different spectral features and can be used to complementarily characterize the organization of fast ripple oscillations.
A
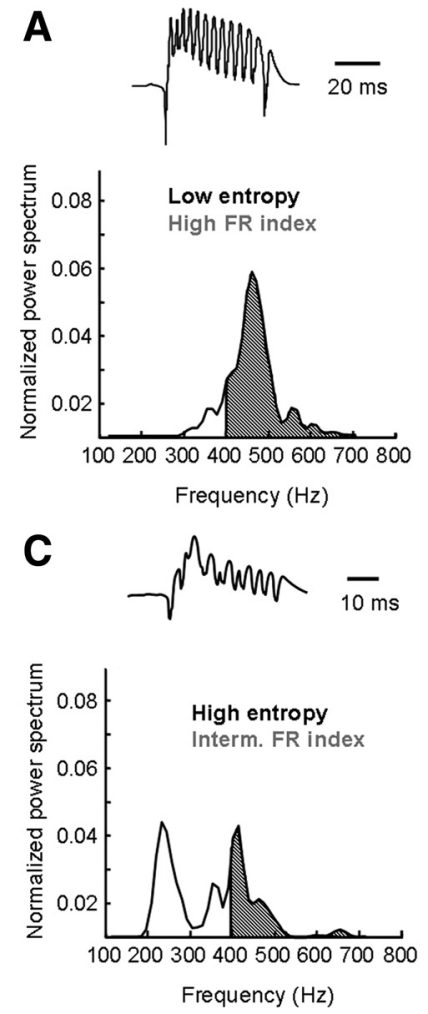

B
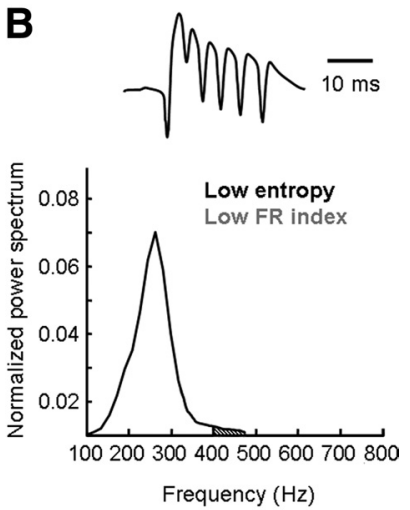

D

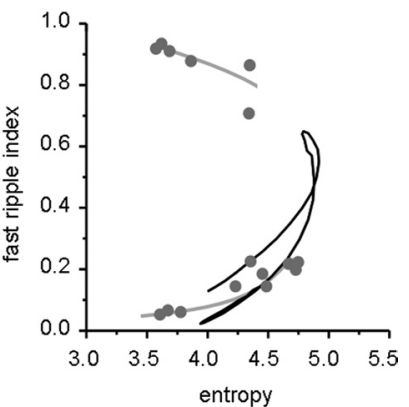

Figure 4. Spectral features of pure and emergent fast ripple oscillations. Schematic diagram illustrating the relationship between the spectral entropy and the FR index for pure and emergent fast ripple oscillations. Each panel show a representative waveform of the fast ripple oscillation and the corresponding normalized power spectrum, from where the spectral entropy and FR index are obtained. $\boldsymbol{A}, \boldsymbol{B}$, Pure fast ripples exhibit a single spectral peak that reflects in-phase cellular firing of similar frequency. Consequently, their power spectrum has low entropy values. Pure fast ripples with significant spectral components faster than $400 \mathrm{~Hz}$ (shadowed region) will exhibit high values of the FR index $(\boldsymbol{A})$, while slower pure fast ripples will exhibit low values of the FR index. C, Emergent fast ripples are characterized by multipeaked spectra of high entropy values. Typically, emergent spectral peaks significantly contribute to the upper fast ripple band giving intermediate values of the FR index. Hence, the major spectral features of pure and emergent fast ripples can be quantified using both the spectral entropy and the FR index. $\boldsymbol{D}$, Plot of the spectral entropy against the fast ripple index for the cluster lag simulations shown in Figure $3 B$ (black) and for simulations of pure oscillations shown in Figure $1 D$ (gray).

Plotting these indexes against each other for each simulation of cluster delays shown in Figure $3 B$ depicted a characteristic curve for emergent oscillations (Fig. $4 D$, black trace). This curve shows that for networks of neurons bursting out-of-phase at low frequencies, emergent oscillations (higher entropy) tend to increase the relative spectral power at the upper fast ripple band (higher FR index). Thus, the emergent regime is characterized by a positive correlation between the entropy and the FR index, upper bounded by the maximum entropy, which represents noisy emergent oscillations. In contrast, for pure oscillations representing in-phase firing at different frequencies shown in Figure $1 D$, the relationship between these indices depended on the frequency. In-phase firing at low frequencies resulted in spectra with low entropy and low FR index (Fig. $4 D$, lower part of the gray curve and dots), while for frequencies approaching the upper fast ripple band, high FR indexes were obtained (Fig. $4 D$, upper part of the gray curve and dots). In this case, for faster pure oscillations (higher FR index), the spectral power tended to concentrate at the upper fast ripple band, necessarily lowering the spectral entropy. Thus a regime of negative correlation between the FR index and the 
A1

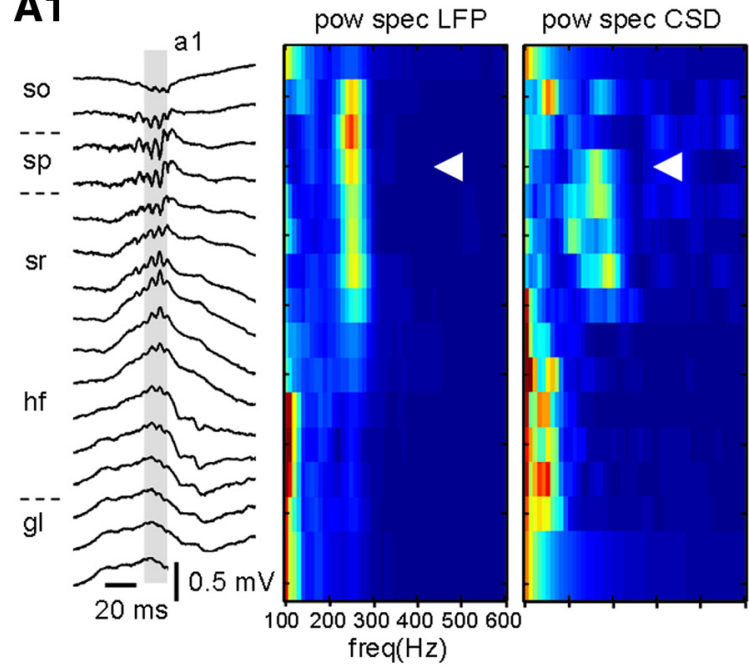

B

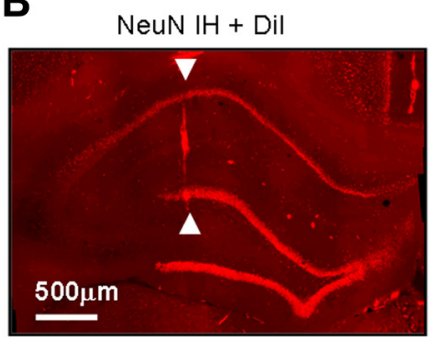

C

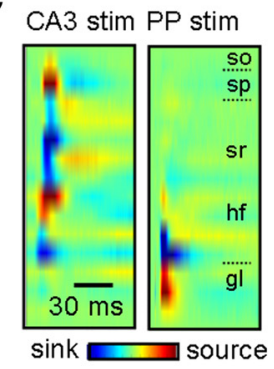

A2

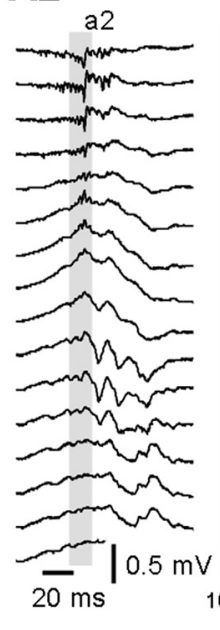

pow spec LFP

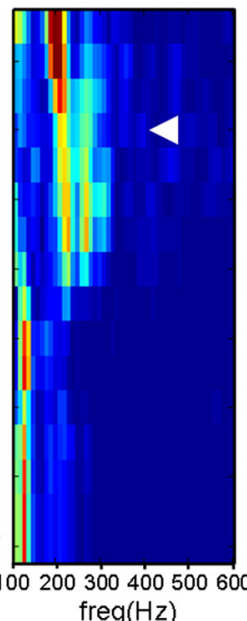

pow spec CSD
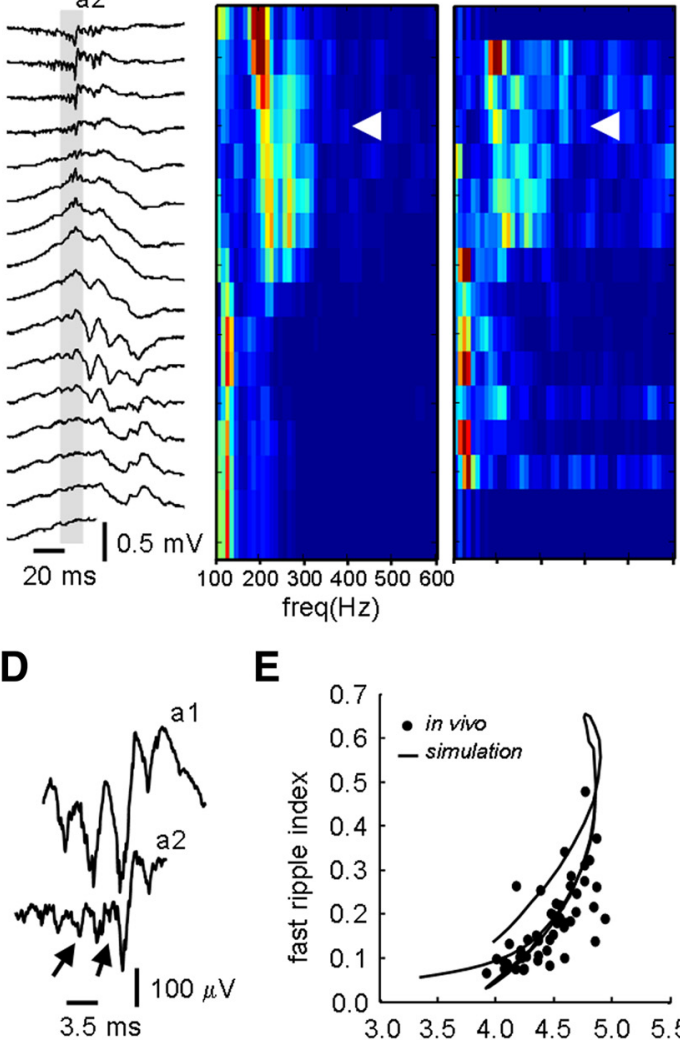

E

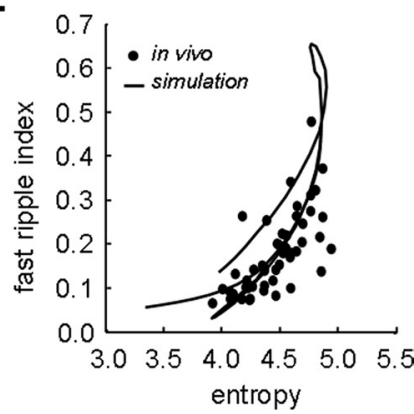

Figure 5. Spontaneous fast ripples in vivo. A1, A2, Two consecutive spontaneous fast ripple events recorded in vivo with 16-channel silicon probes. The electrode position alongside the different strata is indicated. The power spectrum from the LFPs and from the CSD profiles at each channel is plotted in a color-coded scale from zero to maximum. White arrowheads indicate the stratum pyramidale. $\boldsymbol{B}$, Immunostaining $(\mathrm{IH})$ against the neuronal marker NeuN. Note cell loss in the CA3 and hilar regions and the probe track marked with Dil (between arrowheads). See supplemental Figure $1 E$ (available at www.jneurosci.org as supplemental material) for an enlarged view. C, CSD profiles of the responses to subthreshold contralateral CA3 and perforant path (PP) stimulation. $\boldsymbol{D}$, Details of the LFP signals recorded at the stratum pyramidale in panels $\boldsymbol{A} 1$ and $\boldsymbol{A 2}$ (shadowed regions a1 and a2). $\boldsymbol{E}$, Plot of spectral entropy against the fast ripple index for 49 spontaneous events recorded from 6 rats. Note the similarity with simulations (black trace).

entropy would help to distinguish whether fast ripples in the upper frequency band $(400-800 \mathrm{~Hz})$ reflect neuronal firing occurring at such a high-frequency rate or are all emergent components.

In synthesis, our simulation results suggest that fast ripples at the population level can result from two dynamic regimes at the cellular level: (1) precise in-phase cellular bursting, leading to pure oscillations, or (2) out-of-phase cellular bursting in a range of lags within the oscillatory cycle, leading to emergent oscillations. Emergent oscillations in the upper fast ripple band arising from out-of-phase firing at lower frequencies will have spectral entropy and FR indexes positively correlated. In contrast, oscillations resulting from neuronal firing at the upper fast ripple band will exhibit higher FR indices negatively correlated with the spectral entropy. We will use these features of the relationship between the spectral entropy and the FR index to examine fast ripple dynamics from experimental data obtained in vivo.

Spontaneous fast ripples in the epileptic hippocampus in vivo To explore the spectral organization of fast ripples in the epileptic hippocampus, we used unilateral intrahippocampal kainic acidtreated epileptic rats (Bragin et al., 1999) and the urethaneanesthetized preparation ( $n=14$ rats). We obtained multisite recordings of local field potentials from the dorsal hippocampus ipsilateral to kainic acid injections using silicon probes of 16 electrodes interspaced at $100 \mu \mathrm{m}$.
Laminar recordings from these sites revealed spontaneous fast ripples that occurred in the CA1 area of 6 of 14 rats (Fig. 5A1, left). As previously described in non-anesthetized rats (Bragin et al., 2007), fast ripples occurred in association with sharp waves of $200-800 \mu \mathrm{V}$ in the stratum radiatum and occasionally coincided with slower oscillations in the dentate gyrus (Fig. 5A2, left). Immunostaining against the neuronal marker NeuN revealed substantial neuronal loss in the hilus and CA3 regions of the injected hippocampus of all rats examined (Fig. 5B; enlarged image shown in supplemental Fig. $1 E$, available at www.jneurosci.org as supplemental material). CSD analysis of the responses to contralateral subthreshold CA3 stimulation and ipsilateral perforant path stimulation helped to localize the different hippocampal strata (Fig. 5C).

The spatial spectral distribution of spontaneous fast ripple oscillations suggested that they were constrained to the CA1 region covering the strata oriens, pyramidale, and radiatum (Fig. $5 A 1$, middle). Power spectrum analysis of the CSD responses confirmed this idea (Fig. 5A1, right). The frequency content of these oscillations varied substantially from event to event. In Figure $5 A 2$, a consecutive event to that shown in $A 1$ exhibited multiple high-frequency peaks, not strictly harmonics. Detailed examination of ripple cycles at the stratum pyramidale of events shown in $A 1$ and $A 2$ highlighted multiple population spikes breaking down as faster ripples (Fig. $5 D$ ). A positive correlation 
A1

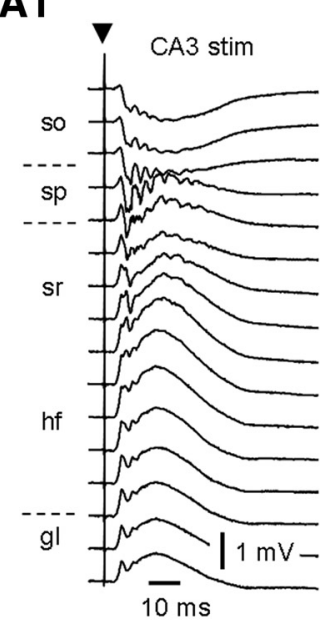

B1

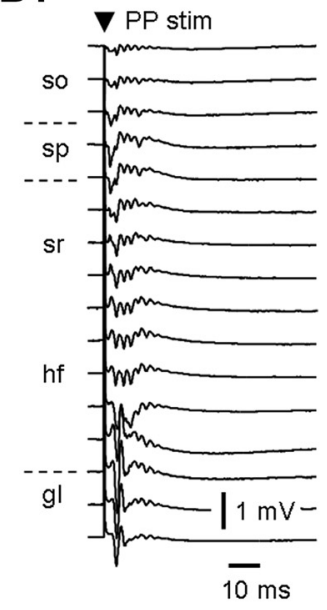

A2

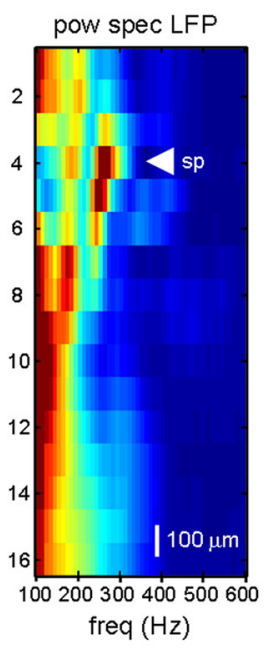

B2

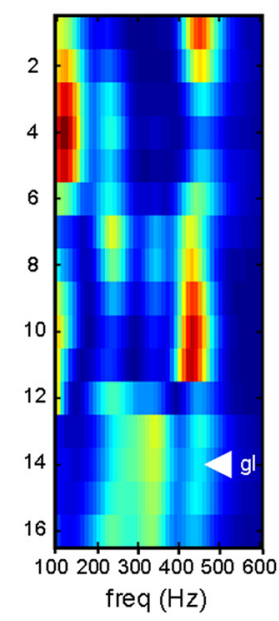

C1

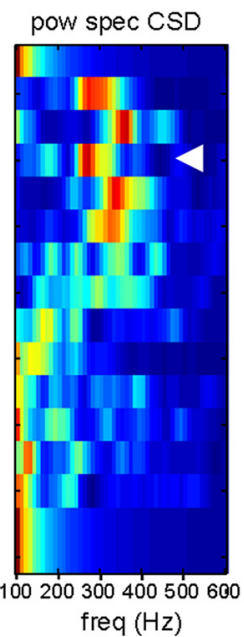

D

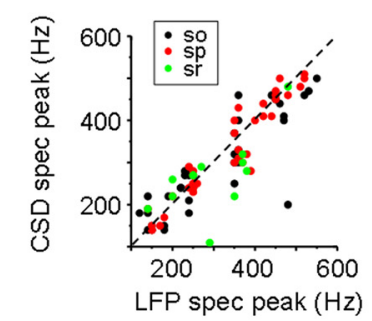

C2
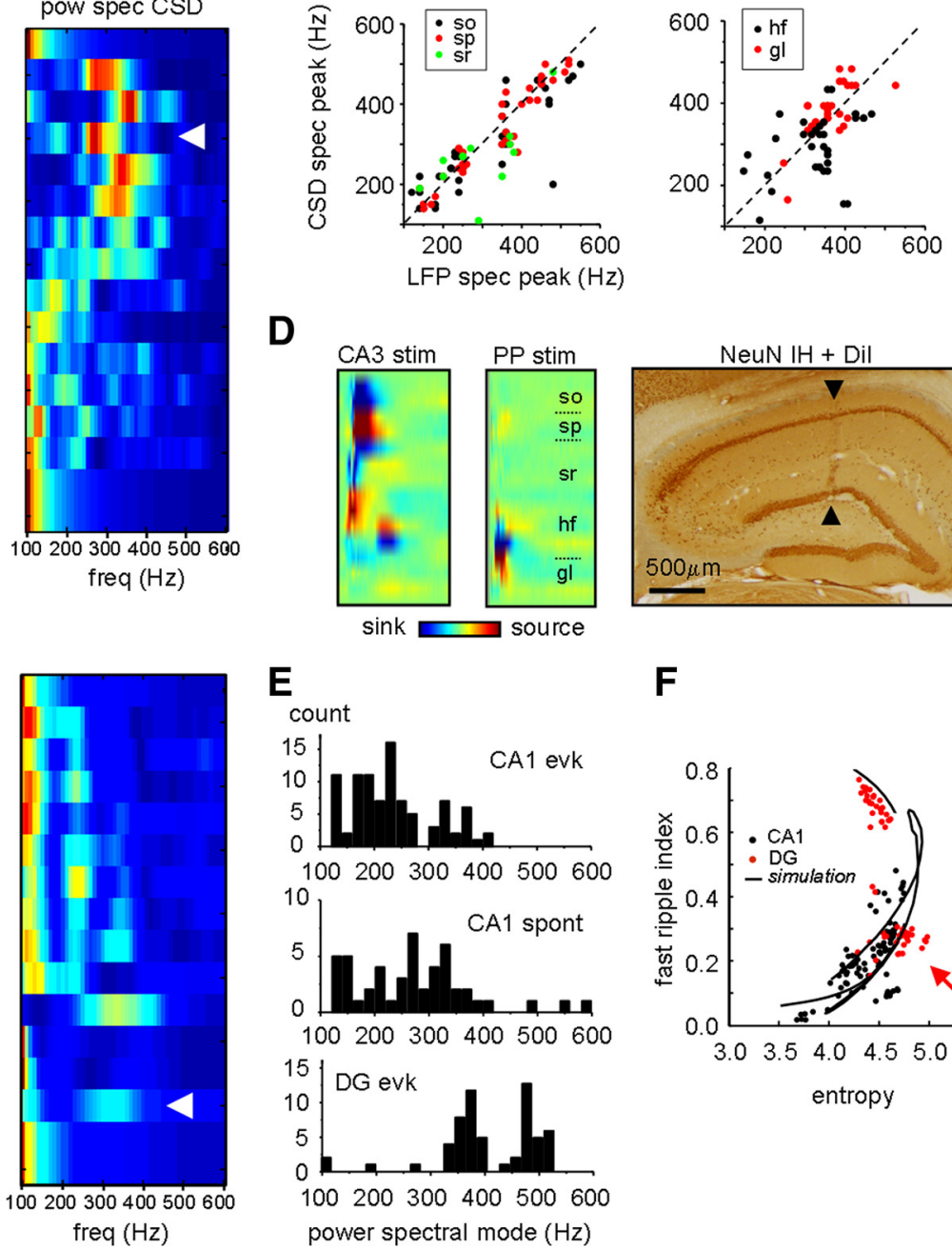

E
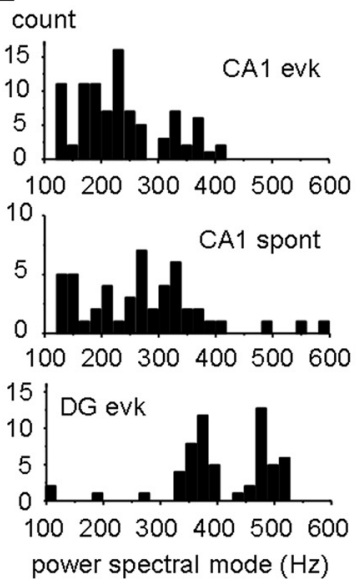

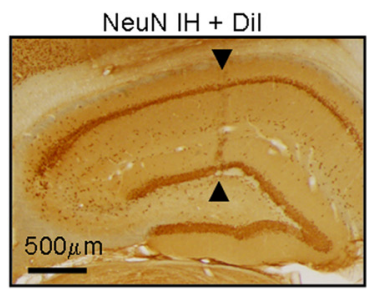

$\mathbf{F}$

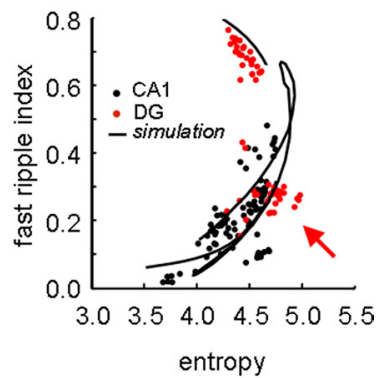

Figure 6. Stimulation-evoked fast ripples in vivo. A1, Local field potential responses to contralateral CA3 stimulation (arrowhead). A2, The power spectrum from the LFPs (left) and from the CSD (right) profiles at each channel is plotted in a color-coded scale from zero to maximum (see supplemental Fig. 4, available at www.jneurosci.org as supplemental material, for the CSD profiles). White arrowheads indicate the stratum pyramidale with similar spectral response between the LFP and the CSD spectra. B1, Laminar responses to ipsilateral perforant path (PP) stimulation (arrowhead). B2, Power spectra obtained from the LFP and the CSD profiles (for the CSD profiles, see supplemental Fig. 4, available at www.jneurosci.org as supplemental material). White arrowheads indicate the granule cell layer. C1, Correspondence between the spectral peaks obtained from the LFP and from the CSD profiles at the strata oriens (so), pyramidale (sp), and radiatum (sr) of the CA1 region upon contralateral CA3 stimulation. C2, Same representation for the profiles obtained at the hippocampal fissure (hf) and granule layer ( $\mathrm{gl}$ ) upon PP stimulation. D, Subthreshold CSD profiles and NeuN immunostaining $(\mathrm{IH})$ for the experiment shown in $\boldsymbol{A}$ and $\boldsymbol{B}$. See supplemental Figure $1 \boldsymbol{F}$ (available at www.jneurosci.org as supplemental material) for an enlarged view of the NeuN section. The probe track is indicated by arrowheads. $\boldsymbol{E}$, Distribution of the most probable frequency peak (mode) from stimulation-evoked (CA1 evk) and spontaneous (CA1 spont) CA1 oscillations and for the dentate gyrus stimulation-evoked events (DG evk). $\boldsymbol{F}$, Plot of spectral entropy against the fast ripple index from 91 stimulation-evoked events in the (A1 region (black) and from 60 stimulationevoked events in the DG (red). Red arrow indicates red points distributed in the lower part of the curve.

between the spectral entropy and the FR index for all events recorded at the stratum pyramidale satisfied the predicted relationship for fast ripples at higher frequencies arising from out-of-phase firing at lower frequencies ( $r=0.66, p<0.001 ; n=49$ events from 6 rats) (Fig. $5 E$ ). This suggests that CA1 fast ripples like the ones shown in Fig. 5A2 might follow emergent dynamics.

\section{Stimulation-evoked fast ripples in vivo}

Spontaneous fast ripples were infrequent under urethane anesthesia. We therefore used suprathreshold stimulation of both the contralateral CA3 and the perforant pathway to evoke them in the hippocampus ipsilateral to the kainic acid injection, as previously described in non-anesthetized rats (Bragin et al., 2002).

Stimulation of the contralateral CA3 evoked fast ripple events with 5-10 ms latency, similar to those recorded spontaneously although of higher amplitudes $(400-1200 \mu \mathrm{V}, n=8$ rats) (Fig.
$6 A 1)$. The power spectrum of the local field potentials and the CSD responses confirmed the presence of fast ripples at the stratum pyramidale of CA1 (Fig. 6A2, arrowhead; supplemental Fig. $4 A$, available at www.jneurosci.org as supplemental material). A good correlation ( $r=0.95, p<0.0001 ; 25$ events from 4 rats) between spectral peaks from the field potential and the CSD profiles supports the idea that fast ripples evoked in CA1 by contralateral CA3 stimulation represent local events (Fig. 6C1).

In contrast, perforant path stimulation resulted in broadly distributed fast ripple events with peaks at $300-600 \mathrm{~Hz}$ frequencies and large amplitudes ( $1-5 \mathrm{mV}, n=7$ rats) (Fig. 6 B1). Short latencies of the order of 2.2-3.5 ms suggested that these fast ripple events resulted from direct activation of granule cells. In this example, electrodes within the dentate gyrus sampled fast ripples exhibiting two spectral peaks, whereas only the highest peak was detected by electrodes far away in the hippocampal fissure and 

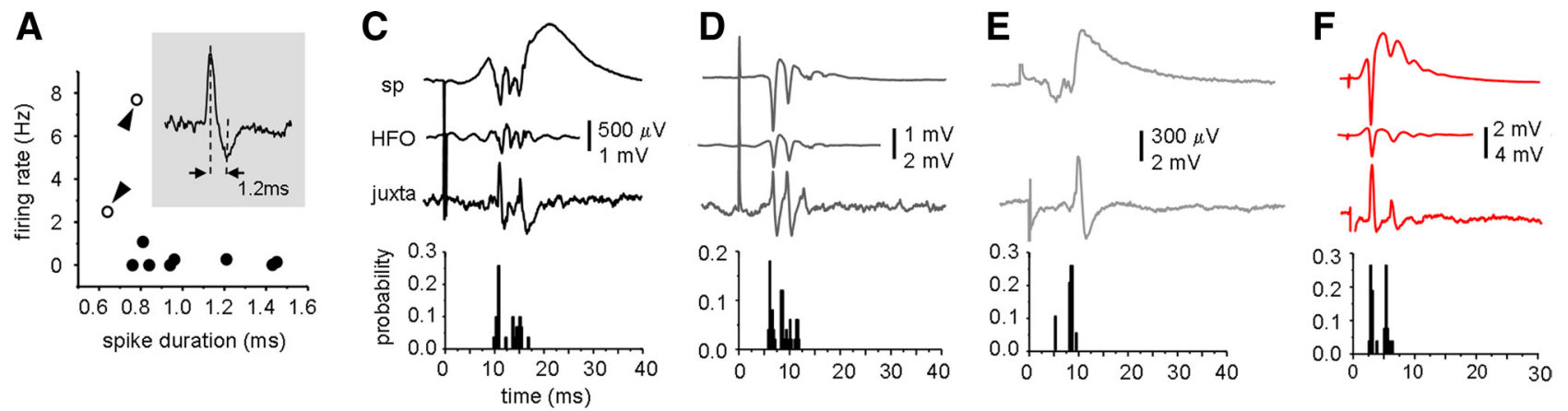
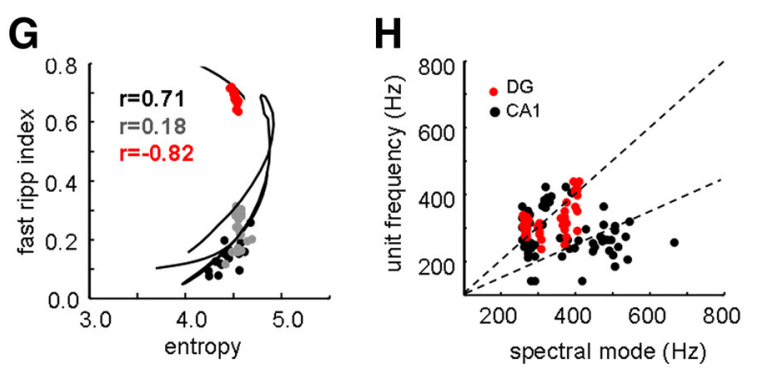

Figure 7. Juxtacellular recordings in vivo. A, Plot of the spike duration versus the firing rate for CA1 unit classification as pyramidal cells (black) and putative interneurons (open dots). The inset illustrates spike width estimation. $\boldsymbol{B}$, Spontaneous firing patterns from units shown as color coded in $\boldsymbol{C}$ to $\boldsymbol{F}$. The black, gray, and light gray traces correspond to $\boldsymbol{C A} 1$ units shown in $\boldsymbol{C}-\boldsymbol{E}$, respectively. The red trace correspond to the unit at the dentate gyrus (DG) shown in $\boldsymbol{F}$. C, Representative response of CA1 units to contralateral CA3 stimulation seen in $4 / 8$ units. Local field potential recording at the stratum pyramidale (SP) and the associated high-frequency oscillations (HF0) are shown, together with the firing histogram. $D, 0$ ne CA1 neuron responded with bursts that were synchronous with the field potential recording. $\boldsymbol{E}$, Three of eight CA1 neurons fired single spikes in response to contralateral CA3 stimulation. In these cases, the oscillatory field potential component was not clear. $\boldsymbol{F}$, Representative response seen in 5/5 DG units to perforant path stimulation. $\mathbf{G}$, Plot of the spectral entropy against the fast ripple index for the field potential oscillations of experiments shown in $\boldsymbol{C}, \boldsymbol{D}$, and $\boldsymbol{F}$, with similar colors. The $r$ values of a Pearson correlation are shown for each case. Lines correspond to simulation prediction. $\boldsymbol{H}$, Unit firing frequency is plotted against the spectral peaks of the nearby fast ripple oscillations ( $250-800 \mathrm{~Hz}$ ) for CA1 (black) and DG (red). An identity discontinuous line is shown to illustrate the dependence of neuronal firing rate on oscillatory frequency. A second discontinuous line indicates the harmonic behavior (frequency $=0.5 \times$ spectral mode).

the stratum radiatum (Fig. 6B2, left spectrum). However, the CSD analysis showed that they were not locally generated (Fig. $6 B 2$, right spectrum; supplemental Fig. $4 B$, available at www. jneurosci.org as supplemental material). The nonlocal character of these oscillations was reflected in the poor correlation between spectral peaks from the field potentials and from the CSD profiles at the hippocampal fissure $(r=0.39, p=0.02 ; 33$ events from 6 rats) (Fig. 6C2). Instead, this correlation was maximal at the granular cell layer $(r=0.74, p<0.0001)$ (Fig. 6C2).

To examine the frequency components of field potential oscillations, we calculated the spectral mode, i.e., the most probable frequency peak of the spectral distribution. The spectral modes of stimulation-evoked fast ripples (91 events from 7 rats) and spontaneous fast ripples ( 49 events from 6 rats) in CA1 stratum pyramidale were similar, with values in the range of $100-400 \mathrm{~Hz}$ (Fig. $6 E$ ). Although we found no spontaneous fast ripples comparable to the stimulation-evoked fast ripples in the dentate gyrus under urethane, similar events has been described in the non-anesthetized preparation (Bragin et al., 2002). To avoid contamination from propagated signals, we used the CSD profiles at the granular layer to estimate the spectral features of perforant path-evoked fast ripples. The most probable frequency peaks were found at the 300-600 $\mathrm{Hz}$ band ( $n=60$ events from 7 rats) (Fig. $6 E$ ).

Plotting the spectral entropy against the FR index for stimulation-evoked data suggested different dynamic regimes of fast ripples in CA1 and in the dentate gyrus (Fig. 6F). Stimulationevoked fast ripples in CA1 displayed a positive correlation between entropy and FR index ( $r=0.63, p<0.001)$ (Fig. $6 F$, black dots), similar to the spontaneous events shown in Figure $5 E$, suggesting that in this region fast ripples at higher frequencies can arise from out-of-phase firing at lower frequencies. In contrast, stimulation- evoked fast ripples in the dentate gyrus displayed high values of FR index that were negatively correlated with the entropy $(r=$ $-0.67, p<0.001$ ) (Fig. $6 F$, red dots), suggesting that they represent oscillations due to neuronal bursting at high-frequency rates. There were cases at intermediate values of FR index that remained ambiguous (Fig. $6 F$, red arrow). Single-cell recordings were then required to fully understand the underlying dynamic.

\section{Juxtacellular recordings from CA1 and dentate gyrus cells in vivo}

Single-cell activity was recorded juxtacellularly using glass pipettes and simultaneous 16-channel silicon probes guided by extracellular stimulation. Most neurons recorded in the CA1 stratum pyramidale exhibited features of pyramidal cells ( 8 of 10 cells from 6 rats), including low spontaneous firing rate $(<1.1$ $\mathrm{Hz}$ ) and spike duration of 0.7-1.5 ms (Fig. 7A) (Ranck, 1973). Two ambiguous neurons with high spontaneous firing rates and short spike durations were not included in the analysis (Fig. 7A, arrowheads). While some overlap has been described in similar parameters obtained from extracellular recordings of pyramidal cells and interneurons, complex-spike bursts appear to be a pyramidal-cell-specific pattern (Fig. 7B) (Ranck, 1973; Csicsvari et al., 1999). We found the first peak of the firing autocorrelogram to be consistent with a CA1 pyramidal-like unit in $5 / 8$ cells $(13.4 \pm 6.8 \mathrm{~ms})$. The remaining three cells did not fire complexspike bursts, but they were classified as pyramids according to large spike duration and low firing rate.

Most CA1 pyramidal cells responded with bursts to contralateral CA3 stimulation (5/8), confirming that fast ripples involve abnormal burst firing. In 4 of these 5 units (Fig. $7 C$ ), concurrent field potential oscillations recorded at the pyramidal layer exhib- 
ited a positive correlation between entropy and FR index, consistent with emergent dynamics (Fig. 7G, black dots). In these cases, pyramidal neurons had intraburst firing rates of $251 \pm 36 \mathrm{~Hz}$ ( $n=4$ units). The individual spikes aligned with preferred field potential cycles with $1.23 \pm 0.18 \mathrm{~ms}$ precision (104 spikes from 4 units). However, variability in the number of spikes from 3 to 1 per event and jumps between cycles within each event were seen, as captured by firing histograms (Fig. 7C, bottom). This dynamic is consistent with the clustering organization observed in our simulations. In one unit from one rat, individual events exhibiting pure oscillations up to $360 \mathrm{~Hz}$ were detected in the nearby field potential and the cell followed that frequency with some variability in the number of spikes emitted (Fig. 7D). The remaining 3 of 8 CA1 pyramidal neurons fired single spikes (Fig. $7 E$ ). The nearby field potential oscillations were not clear in these cases. Overall, there were no correlation between the intraburst frequency from CA1 single-cells and the spectral peaks of the field potential fast ripple oscillation $(r=-0.11, p=0.37, n=65$ events, 5 cells from 4 rats) (Fig. $7 H$, black dots), suggesting that fast ripples in the $\mathrm{CA} 1$ region mostly reflect emergent oscillations due to out-of-phase firing.

In contrast, juxtacellular recordings from granule cells confirmed that fast ripples in the dentate gyrus mostly reflected pure oscillations due to synchronous bursting. Granule cells rarely fired spontaneously between events and had spike durations similar to pyramidal cells $(0.8-1.5 \mathrm{~ms}, n=5$; Ranck, 1973$)$. Perforant path stimulation induced bursting responses in all 5 units recorded from 3 rats with intraburst frequency of $320 \pm 49 \mathrm{~Hz}$, slightly higher than CA1 cells (Fig. $7 F$ ). In all 3 rats, nearby field potential oscillations, recorded at the granular layer, occurred at the $250-400 \mathrm{~Hz}$ band, and individual spikes had a firing precision of $0.77 \pm 0.50 \mathrm{~ms}$ (103 spikes from 5 units). A negative correlation between the entropy and the FR index of field potential oscillations suggested a different mechanism compared to CA1 (Fig. 7G, red dots). Indeed, single-cell frequency was positively correlated with the spectral peaks of the field potential fast ripple oscillation, in contrast to CA1 cells $(r=0.56, p<0.0001$; $n=49$ events from 5 cells) (Fig. $7 H$, red dots). This correlation occurred within a limited frequency range of $250-400 \mathrm{~Hz}$. This suggests that in the dentate gyrus, stimulation-evoked fast ripples mostly represent pure oscillations resulting from synchronous neuronal firing at $250-400 \mathrm{~Hz}$.

\section{Discussion}

Our results suggest that fast ripples can arise both from in-phase firing and from out-of-phase firing in the epileptic hippocampus. In-phase firing results from pathological synchronous bursting, producing pure oscillations that can reach frequencies up to 300 $\mathrm{Hz}$ in the CAl and up to $400 \mathrm{~Hz}$ in the dentate gyrus. The organization of out-of-phase firing is determined by firing delays between neuronal clusters in the range of $1 / 2$ to $\sim 3 / 4$ of the intraburst cycle, producing emergent oscillations at higher frequencies from slower-discharging cells. These two firing regimes emerge dynamically and interchangeably on an event-to-event basis in the epileptic hippocampus.

Oscillatory field potentials with a wide range of frequencies have been observed and studied in the normal and pathological hippocampus, from ultraslow oscillations, through theta, beta, and gamma rhythms, to physiological ripples in the $100-200 \mathrm{~Hz}$ band (Buzsáki and Draguhn, 2004). Fast ripples, with a frequency of $250-800 \mathrm{~Hz}$, have been identified in the epileptic hippocampus of rats and humans $\sim 10$ years ago, but their cellular basis remains controversial.
There is debate regarding whether individual neuronal firing follows all the frequency range of fast ripples, i.e., $250-800 \mathrm{~Hz}$ (Staley, 2007; Engel et al., 2009). The current doctrine is that fast ripples reflect pathological hypersynchronous bursting from principal neurons (Bragin et al., 2000). In classical experiments with penicillin foci, most cells burst synchronously during population discharges (Matsumoto and Ajmone Marsan, 1964). Thus, extracellular dipoles formed by precise bursting of paralleloriented pyramidal cells summate to produce repetitive population spikes that reflect individual neuronal firing. However, such a high level of synchrony and bursting is not reproduced in other focal models and is controversial in human temporal lobe epilepsy (Wyler et al., 1975; Colder et al., 1996).

Our juxtacellular data confirmed that fast ripples reflect not only pure oscillations due to population spikes from principal cells discharging synchronously at $100-400 \mathrm{~Hz}$, but also emergent oscillations. Under certain conditions, neuronal bursting was synchronous and high-frequency oscillations perfectly matched single-cell behavior. This seemed to be often the case in the dentate gyrus but not in most CA1 neurons in vivo or CA3 cells in vitro (Foffani et al., 2007), where individual neurons typically fired slower than $300 \mathrm{~Hz}$ and faster ripples were observed in the field potential. Possible mechanisms underlying this difference include different intrinsic neuronal firing properties and synaptic circuitry between the epileptic CA1 and dentate regions (Esclapez et al., 1999; Nägerl et al., 2000; Kobayashi and Buckmaster, 2003).

Under urethane anesthesia, some spontaneous pure oscillations in the CA1 region of epileptic rats occurred at $100-300 \mathrm{~Hz}$, suggesting that oscillations at that frequency reflect pathological synchronous bursting. Notably, these oscillations might contrast with physiological ripples $(100-200 \mathrm{~Hz})$ in that they involve firing from a larger number of neurons, and that in normal ripples, pyramidal cells burst less reliably (Csicsvari et al., 1999, 2000; Harris et al., 2001), with a main contribution of inhibitory field potentials (Ylinen et al., 1995). Interneuronal firing is not proposed to contribute directly to fast ripples, although the postsynaptic role of inhibitory currents remains unclear. Recent work suggests that the contribution of inhibitory currents to the extracellular field potential during population discharges is masked by increased pyramidal cell firing (Trevelyan, 2009). Indeed, antagonists of $\mathrm{GABA}_{\mathrm{A}}$ receptors have no effect on fast ripples in vitro (Maier et al., 2003), similar to high-frequency oscillations associated with sensory evoked potentials (Jones and Barth, 2002; Staba et al., 2004). Instead, synchronous bursting in the hippocampus seems to require disinhibition (Wong et al., 1979; Miles and Wong, 1987), or prolonged periods of neuronal silence followed by dendritic excitation (Kamondi et al., 1998; Harris et al., 2001). These mechanisms need to be further explored to better understand the role of interneuronal firing in fast ripples (Spampanato and Mody, 2007) and the relationship between normal ripples and pathological forms of high-frequency oscillations.

Our data suggest that most events containing fast ripples $>400 \mathrm{~Hz}$ resulted from emergent oscillations. In every experiment, there was large variability from event to event with spectral fluctuations between low- and high-frequency bands. This is consistent with reports of pathological high-frequency oscillations in the ripple band being typically recorded intermixed with fast ripples (Bragin et al., 1999; Staba et al., 2002; Worrell et al., 2008). Our computational simulations suggested that the emergent oscillations are built upon functional clustering of bursting cells and emerge at a range of synaptic strengths and noise levels. Similar clustering was previously described in a detailed mathe- 
matical analysis of a simplified Traub model with all-to-all connections (Pinsky, 1995), and in other models of excitatory neurons with specific coupling parameters (Li et al., 2003). Such clustering is evident experimentally in the appearance of emergent population spikes. Cluster delays of the order of 2-4 ms revealed to be optimal for emergent oscillations. These periods result from firing delays between cells being recruited after polysynaptic propagation and due to spike-generation dynamics (Miles et al., 1988; Fricker and Miles, 2000). The role of neuronal delays has been described in models of fast oscillations from 40 to $200 \mathrm{~Hz}$ exhibiting a rich repertoire of emergent behaviors (Brunel and Wang, 2003; Roxin et al., 2005). Importantly, spikes separated by $0.5-1.5 \mathrm{~ms}$ would contribute together to the same cycle, reflecting the temporal scales required to generate population ripples (Andersen et al., 1971; Barth, 2003). That explains why both precise and imprecise firing are claimed to be responsible for fast ripples (Dzhala and Staley, 2004; Foffani et al., 2007). Precise neuronal discharges synchronize in-phase to build pure oscillations of similar frequencies (Dzhala and Staley, 2004), whereas imprecise firing breaks down into clusters, leading to emergent oscillations at higher frequencies (Foffani et al., 2007). One corollary of these two studies, together with the current work, is that a network of heterogeneous neurons would rarely be synchronized in-phase and emergent oscillations would dominate the field potential spectrum. This is the case of the effect of synaptic noise in our simulations, which tends to reduce the reliability of interspike intervals and the timing of neuronal firing.

From a clinical perspective, it has been suggested that fast ripples could have a diagnostic value to identify epileptogenic regions (Bragin et al., 2000; Staba et al., 2007; Worrell et al., 2008). However, to identify pathological fast ripples in clinical settings and to understand the underlying mechanisms based only on their frequency content is not a straightforward task (Engel et al., 2009). Our study suggests that the dynamical behavior of high-frequency oscillations-as evaluated from the spectral properties of single events and from their trial-totrial variability - can provide useful information about the underlying pathophysiology. Such information could be helpful for improving the classification of diverse forms of high-frequency oscillations (Blanco et al., 2010).

In summary, our results support the idea that fast ripples in the epileptic hippocampus alternate randomly between in-phase and out-of-phase neuronal firing. They do not reflect single-cell behavior per se, as perfect synchronization of individual neurons is just one of the several ways cells become organized at the population level during shared activation. Therefore, emergent oscillations reflect the functional dynamic of the circuit, and any process promoting firing disorganization would favor their appearance. This would include neuronal loss (Staba et al., 2007), but also elevated synaptic noise (Esclapez et al., 1999; Foffani et al., 2007), circuit reorganization (Nadler et al., 1980; Kobayashi and Buckmaster, 2003; Shao and Dudek, 2005), or different channelopathies affecting intrinsic firing patterns (Bernard et al., 2004).

\section{References}

Adrian E (1935) Discharge frequencies in the cerebral and cerebellar cortex. Proc Phys Soc 83:32-33.

Andersen P, Bliss TVP, Skrede KK (1971) Unit analysis of hippocampal population spikes. Exp Brain Res 13:208-221.

Barth DS (2003) Submillisecond synchronization of fast electrical oscillations in neocortex. J Neurosci 23:2502-2510.

Beenhakker MP, Huguenard JR (2009) Neurons that fire together also con- spire together: is normal sleep circuitry hijacked to generate epilepsy? Neuron 62:612-632.

Bernard C, Anderson A, Becker A, Poolos NP, Beck H, Johnston D (2004) Acquired dendritic channelopathy in temporal lobe epilepsy. Science 305:532-535.

Bikson M, Fox JE, Jefferys JG (2003) Neuronal aggregate formation underlies spatiotemporal dynamics of nonsynaptic seizure initiation. J Neurophysiol 89:2330-2333.

Blanco JA, Stead M, Krieger A, Viventi J, Marsh WR, Lee KH, Worrell GA, Litt B (2010) Unsupervised classification of high-frequency oscillations in human neocortical epilepsy and control patients. J Neurophysiol. J Neurophysiol 104:2900-2912.

Bragin A, Engel J Jr, Wilson CL, Fried I, Mathern GW (1999) Hippocampal and entorhinal cortex high-frequency oscillations $(100-500 \mathrm{~Hz})$ in human epileptic brain and in kainic acid-treated rats with chronic seizures. Epilepsia 40:127-137.

Bragin A, Wilson CL, Engel J Jr (2000) Chronic epileptogenesis requires development of a network of pathologically interconnected neuron clusters: a hypothesis. Epilepsia 41 [Suppl 6]:S144-S152.

Bragin A, Mody I, Wilson CL, Engel J Jr (2002) Local generation of fast ripples in epileptic brain. J Neurosci 22:2012-2021.

Bragin A, Wilson CL, Engel J Jr (2007) Voltage depth profiles of highfrequency oscillations after kainic acid-induced status epilepticus. Epilepsia 48:35-40.

Brunel N, Wang XJ (2003) What determines the frequency of fast network oscillations with irregular neural discharges? I. Synaptic dynamics and excitation-inhibition balance. J Neurophysiol 90:415-430.

Buzsáki G, Draguhn A (2004) Neuronal oscillations in cortical networks. Science 304:1926-1929.

Cheron G, Servais L, Wagstaff J, Dan B (2005) Fast cerebellar oscillation associated with ataxia in a mouse model of Angelman syndrome. Neuroscience 130:631-637.

Colder BW, Wilson CL, Frysinger RC, Chao LC, Harper RM, Engel J Jr (1996) Neuronal synchrony in relation to burst discharge in epileptic human temporal lobes. J Neurophysiol 75:2496-2508.

Csicsvari J, Hirase H, Czurkó A, Mamiya A, Buzsáki G (1999) Oscillatory coupling of hippocampal pyramidal cells and interneurons in the behaving rat. J Neurosci 19:274-287.

Csicsvari J, Hirase H, Mamiya A, Buzsáki G (2000) Ensemble patterns of hippocampal CA3-CA1 neurons during sharp wave-associated population events. Neuron 28:585-594.

Draguhn A, Traub RD, Schmitz D, Jefferys JG (1998) Electrical coupling underlies high-frequency oscillations in the hippocampus in vitro. Nature 394:189-192.

Dzhala VI, Staley KJ (2004) Mechanisms of fast ripples in the hippocampus. J Neurosci 24:8896-8906.

Engel J Jr, Bragin A, Staba R, Mody I (2009) High-frequency oscillations: what is normal and what is not? Epilepsia 50:598-604.

Esclapez M, Hirsch JC, Ben-Ari Y, Bernard C (1999) Newly formed excitatory pathways provide a substrate for hyperexcitability in experimental temporal lobe epilepsy. J Comp Neurol 408:449-460.

Foffani G, Priori A, Egidi M, Rampini P, Tamma F, Caputo E, Moxon KA, Cerutti S, Barbieri S (2003) 300-Hz subthalamic oscillations in Parkinson's disease. Brain 126:2153-2163.

Foffani G, Uzcategui YG, Gal B, Menendez de la Prida L (2007) Reduced spike-timing reliability correlates with the emergence of fast ripples in the rat epileptic hippocampus. Neuron 55:930-941.

Fricker D, Miles R (2000) EPSP amplification and the precision of spike timing in hippocampal neurons. Neuron 28:559-569.

Gobbelé R, Buchner H, Curio G (1998) High-frequency (600 Hz) SEP activities originating in the subcortical and cortical human somatosensory system. Electroencephalogr Clin Neurophysiol 108:182-189.

Harris KD, Hirase H, Leinekugel X, Henze DA, Buzsáki G (2001) Temporal interaction between single spikes and complex spike bursts in hippocampal pyramidal cells. Neuron 32:141-149.

Jiruska P, Finnerty GT, Powell AD, Lofti N, Cmejla R, Jefferys JG (2010) Epileptic high-frequency network activity in a model of non-lesional temporal lobe epilepsy. Brain 133:1380-1390.

Jones MS, Barth DS (1999) Spatiotemporal organization of fast $(>200 \mathrm{~Hz})$ electrical oscillations in rat vibrissa/barrel cortex. J Neurophysiol 82: 1599-1609.

Jones MS, Barth DS (2002) Effects of bicuculline methiodide on fast $(>200$ 
$\mathrm{Hz}$ ) electrical oscillations in rat somatosensory cortex. J Neurophysiol 88:1016-1025.

Kamondi A, Acsády L, Buzsáki G (1998) Dendritic spikes are enhanced by cooperative network activity in the intact hippocampus. J Neurosci 18:3919-3928.

Khosravani H, Pinnegar CR, Mitchell JR, Bardakjian BL, Federico P, Carlen PL (2005) Increased high-frequency oscillations precede in vitro low-Mg seizures. Epilepsia 46:1188-1197.

Kobayashi M, Buckmaster PS (2003) Reduced inhibition of dentate granule cells in a model of temporal lobe epilepsy. J Neurosci 23:2440-2452.

Kreuz T, Chicharro D, Andrzejak RG, Haas JS, Abarbanel HD (2009) Measuring multiple spike train synchrony. J Neurosci Methods 183:287-299.

Li YX, Wang YQ, Miura R (2003) Clustering in small networks of excitatory neurons with heterogeneous coupling strengths. J Comput Neurosci 14:139-159.

López-Azcárate J, Tainta M, Rodríguez-Oroz MC, Valencia M, González R, Guridi J, Iriarte J, Obeso JA, Artieda J, Alegre M (2010) Coupling between beta and high-frequency activity in the human subthalamic nucleus may be a pathophysiological mechanism in Parkinson's disease. J Neurosci 30:6667-6677.

Maier N, Nimmrich V, Draguhn A (2003) Cellular and network mechanisms underlying spontaneous sharp wave-ripple complexes in mouse hippocampal slices. J Physiol 550:873-887.

Malmivuo J, Plonsey R (1995) Bioelectromagnetism—principles and applications of bioelectric and biomagnetic fields. New York: Oxford UP.

Matsumoto H, Ajmone Marsan C (1964) Cortical interictal phenomena in experimental epilepsy: interictal manifestations. Exp Neurol 9:286-304.

Menendez de la Prida L, Huberfeld G, Cohen I, Miles R (2006) Threshold behavior in the initiation of hippocampal population bursts. Neuron 49:131-142.

Middleton SJ, Racca C, Cunningham MO, Traub RD, Monyer H, Knöpfel T, Schofield IS, Jenkins A, Whittington MA (2008) High-frequency network oscillations in cerebellar cortex. Neuron 58:763-774.

Miles R, Wong RK (1983) Single neurones can initiate synchronized population discharge in the hippocampus. Nature 306:371-373.

Miles R, Wong RK (1987) Inhibitory control of local excitatory circuits in the guinea-pig hippocampus. J Physiol 388:611-629.

Miles R, Traub RD, Wong RK (1988) Spread of synchronous firing in longitudinal slices from the CA3 region of the hippocampus. J Neurophysiol 60:1481-1496.

Nadler JV, Perry BW, Cotman CW (1980) Selective reinnervation of hippocampal area CA1 and the fascia dentata after destruction of CA3-CA4 afferents with kainic acid. Brain Res 182:1-9.

Nägerl UV, Mody I, Jeub M, Lie AA, Elger CE, Beck H (2000) Surviving granule cells of the sclerotic human hippocampus have reduced $\mathrm{Ca}^{2+}$ influx because of a loss of calbindin- $\mathrm{D}_{28 \mathrm{k}}$ in temporal lobe epilepsy. J Neurosci 20:1831-1836.

Nguyen DP, Wilson MA, Brown EN, Barbieri R (2009) Measuring instantaneous frequency of local field potential oscillations using the Kalman smoother. J Neurosci Methods 184:365-374.

Pinsky PF (1995) Synchrony and clustering in an excitatory neural network model with intrinsic relaxation kinetics. SIAM J Appl Math 55:220-241.

Racine RJ (1972) Modification of seizure activity by electrical stimulation. II. Motor seizure. Electroencephalogr Clin Neurophysiol 32:281-294.

Ranck JB Jr (1973) Studies on single neurons in dorsal hippocampal formation and septum in unrestrained rats. I. Behavioral correlates and firing repertoires. Exp Neurol 41:461-531.

Richter JP, Behrens CJ, Chakrabarty A, Heinemann U. (2008) Effects of 4 -aminopyridine on sharp wave-ripples in rat hippocampal slices. Neuroreport 19:491-496, 2008 Mar 5.
Roxin A, Brunel N, Hansel D (2005) Role of delays in shaping spatiotemporal dynamics of neuronal activity in large networks. Phys Rev Lett 94 : 238103.

Shao LR, Dudek FE (2004) Increased excitatory synaptic activity and local connectivity of hippocampal CA1 pyramidal cells in rats with kainateinduced epilepsy. J Neurophysiol 92:1366-1373.

Shao LR, Dudek FE (2005) Changes in mIPSCs and sIPSCs after kainate treatment: evidence for loss of inhibitory input to dentate granule cells and possible compensatory responses. J Neurophysiol 94:952-960.

Spampanato J, Mody I (2007) Spike timing of lacunosom-moleculare targeting interneurons and CA3 pyramidal cells during high-frequency network oscillations in vitro. J Neurophysiol 98:96-104.

Staba RJ, Wilson CL, Bragin A, Fried I, Engel J Jr (2002) Quantitative analysis of high-frequency oscillations $(80-500 \mathrm{~Hz})$ recorded in human epileptic hippocampus and entorhinal cortex. J Neurophysiol 88:1743-1752.

Staba RJ, Bergmann PC, Barth DS (2004) Dissociation of slow waves and fast oscillations above $200 \mathrm{~Hz}$ during GABA application in rat somatosensory cortex. J Physiol 561:205-214.

Staba RJ, Frighetto L, Behnke EJ, Mathern GW, Fields T, Bragin A, Ogren J, Fried I, Wilson CL, Engel J Jr (2007) Increased fast ripple to ripple ratios correlate with reduced hippocampal volumes and neuron loss in temporal lobe epilepsy patients. Epilepsia 48:2130-2138.

Staley KJ (2007) Neurons skip a beat during fast ripples. Neuron 55: $828-830$.

Thomson DJ (1982) Spectrum estimation and harmonic analysis. Proc IEEE 70:1055-1096.

Traub RD, Miles R (1991) Neuronal networks of the hippocampus. New York: Cambridge UP.

Traub RD, Wong RK (1982) Cellular mechanism of neuronal synchronization in epilepsy. Science 216:745-747.

Traub RD, Miles R, Wong RK (1989) Model of the origin of rhythmic population oscillations in the hippocampal slice. Science 243:1319-1325.

Traub RD, Wong RK, Miles R, Michelson H (1991) A model of a CA3 hippocampal pyramidal neuron incorporating voltage-clamp data on intrinsic conductances. J Neurophysiol 66:635-650.

Traub RD, Schmitz D, Jefferys JG, Draguhn A (1999) High-frequency population oscillations are predicted to occur in hippocampal pyramidal neuronal networks interconnected by axoaxonal gap junctions. Neuroscience 92:407-426.

Trevelyan AJ (2009) The direct relationship between inhibitory currents and local field potentials. J Neurosci 29:15299-15307.

Urrestarazu E, Chander R, Dubeau F, Gotman J (2007) Interictal highfrequency oscillations $(100-500 \mathrm{~Hz})$ in the intracerebral EEG of epileptic patients. Brain 130:2354-2366.

Varona P, Ibarz JM, López-Aguado L, Herreras O (2000) Macroscopic and subcellular factors shaping population spikes. J Neurophysiol 83:2192-2208.

Wong RK, Prince DA, Basbaum AI (1979) Intradendritic recordings from hippocampal neurons. Proc Natl Acad Sci U S A 76:986-990.

Worrell GA, Gardner AB, Stead SM, Hu S, Goerss S, Cascino GJ, Meyer FB, Marsh R, Litt B (2008) High-frequency oscillations in human temporal lobe: simultaneous microwire and clinical macroelectrode recordings. Brain 131:928-937.

Wyler AR, Fetz EE, Ward AA Jr (1975) Firing patterns of epileptic and normal neurons in the chronic alumina focus in undrugged monkeys during different behavioral states. Brain Res 98:1-20.

Ylinen A, Bragin A, Nádasdy Z, Jandó G, Szabó I, Sik A, Buzsáki G (1995) Sharp wave-associated high-frequency oscillation $(200 \mathrm{~Hz})$ in the intact hippocampus: network and intracellular mechanisms. J Neurosci 15:30-46. 